\title{
Birnbaum Importance based Heuristics for Multi-type Component Assignment Problems
}

Xiaoyan Zhu, Yuqiang Fu

School of Economics and Management, University of Chinese Academy of Sciences, Beijing, China

Tao Yuan, Xinying Wu

Industrial and Systems Engineering Department, Ohio University, Athens, USA

\begin{abstract}
This paper proposes a new type of system reliability design problem, namely, the multi-type component assignment problem (MCAP). The MCAP is to assign more than one type of components to positions in a system with the objective of maximizing system reliability. Each type of components has multiple units, and a unit of a particular type can be assigned to a position of the same type in the system. We present the Birnbaum-importance based local search methods and the Birnbaum-importance based genetic algorithms for addressing the MCAP. The comprehensive numerical experiments are conducted for the $\operatorname{Lin} / \mathrm{Con} / \mathrm{k} / n: \mathrm{F}(\mathrm{G})$ systems with multiple types of components and four cases of component reliability. The computational results demonstrate the efficiencies of the heuristics, highlighting the methods of dealing with multiple types of components. It shows that the Birnbaum importance in designing the algorithms is promising because of its effectiveness.
\end{abstract}

Keywords: multi-type component assignment problem; Birnbaum importance; genetic local search; linear consecutive $k$-out-of- $n$ system

\section{Introduction}

It is common that a product or a system may consist of multiple types of components and needs more than one unit for a type of components. For example, the satellite communication system consists of at least several ground communication stations and several connectors in a satellite [1]. The type of a component is defined based on its function in the system; in other words, the components of the same type are functionally exchangeable. Because of the limited budget or different extent of wear or different brands, the functionally exchangeable components may have different quality, ages, conditions, and eventually different reliability.

In this paper, we study a multi-type component assignment problem (MCAP), in which more than one type of components are to be assigned to positions in a system in order to complete the reliability design of the system. For each type, there is a set of functionally exchangeable components to be assigned to a set of positions of the same type in the system. Because the functionally exchangeable components may have different reliability and the positions are generally not in parallel or series, the assignment of these components to the positions in the system is critical and can result in different system reliability. The MCAP is to determine the assignment of components in a system with the objective of maximizing the system reliability. Section 2 presents a mathematical model to describe the MCAP.

If the system has only one type of multiple components, the MCAP becomes a single-type component assignment problem (SCAP), in which all the components in the system are functionally exchangeable and a component can be assigned to any position in the system. The SCAP is to find the optimal assignment of $n$ available components to $n$ positions in a system to maximize the system reliability [2]. The SCAP is the basis for designing some modern complex systems such as computer network systems $[3,4]$, electrical power systems [5], and so on. Motivated by these applications, the SCAP has been extended to multistate network to either optimize the network reliability, which is the probability of

*: Corresponding author

E-mail address: yuan@ohio.edu.

(C) 2016. This manuscript version is made available under the Elsevier user license

http://www.elsevier.com/open-access/userlicense/1.0/ 
transforming a given demand [3], or to maximize the component allocation cost, in which the cost of allocating a component is counted in terms of the arc length [4].

Since a system usually consists of multiple types of components, the MCAP has broader applications than the SCAP in practice. However, to the best of our knowledge, the MCAP has not been defined and studied yet. The SCAP and MCAP is an important subject in the field of system reliability design. In this paper, we first formally define the MCAP. Yao et al. [6] show that the SCAP is NP-hard; thus, the MCAP is NP-hard as an extension of the SCAP. The exact optimal assignment of the MCAP needs extensive efforts and can be obtained by enumeration method for small-size instances. To address the MCAP, we propose local search heuristics and genetic algorithms, all of which use the Birnbaum importance measure (BI).

To address the SCAP, the enumeration method is the only method reported for the exact optimal assignment for small-size instances [2]. Some local search and global search heuristics have been proposed for addressing the SCAP. The randomization method [2], simulated annealing [7], and genetic algorithm [8] are also applied; the latter two methods are only used to solve the SCAP in the $\mathrm{Cir} / \mathrm{Con} / \mathrm{k} / n: \mathrm{F}$ and $\mathrm{G}$ systems. A Cir/Con $/ \mathrm{k} / n$ system is a system of $n$ ordered components that are connected circularly, and the $\operatorname{Cir} / \operatorname{Con} / k / n: \mathrm{F}(\mathrm{G})$ system fails (works) if and only if at least $k$ consecutive components fail (work). To address the extended SCAP on multistate network, Lin and Yeh [3] develop a minimal-cut based genetic algorithm to maximize the network reliability, and Yeh and Lin [4] develop an optimization algorithm, which integrates tabu search and minimal paths. However, these methods do not utilize the importance measures, which have been shown to be able to improve the performance of heuristics $[6,9,10]$.

Importance measures have been widely used in reliability engineering and safety and risk analysis. For recent works, Natvig et al. [11] study the Barlow-Proschan importance measure and Natvig importance measure in a repairable multistate system. Vaurio [12] claim that a criticality importance is appropriate for ranking and optimization in risk-informed decision making. Contini and Matuzas [13] and Vaurio [14] use the BI and other importance measures in fault tree analysis. Espiritu [15] and Bisanovic [16] extend the BI and criticality importance measures for power industry, in which the electricity distribution systems are normally multistate. Over various types of importance measures, the BI is the most fundamental one and has been widely used $[17,18,19]$.

Recently, the BI is used in various heuristics for addressing the SCAP [6,9]. The BI of position $j$ is defined as the probability that the failure of the component in position $j$ results in system failure and can be calculated as [20]

$$
B I(j)=\frac{\partial R\left(p_{1}, p_{2}, \mathbf{K}, p_{n}\right)}{\partial p_{j}}=R\left(p_{1}, \mathbf{K}, p_{j-1}, 1, p_{j+1}, \mathbf{K}, p_{n}\right)-R\left(p_{1}, \mathbf{K}, p_{j-1}, 0, p_{j+1}, \mathbf{K}, p_{n}\right)
$$

where $p_{j}$ denotes the reliability of the component in position $j$ (i.e., the reliability of $\operatorname{position} j$ ) for $j=1$, $2, \ldots, n$, and $R(\cdot)$ denotes the system reliability with the corresponding reliability of positions. From (1), the $\mathrm{BI}$ is to evaluate the relative importance and contribution of a component to system reliability. Thus, a position with a larger BI value intuitively deserves a more reliable component. The BI can be used in heuristics to guide the assignment of components in a system.

There are two groups of the BI-based heuristics for the SCAP: the local search methods and the genetic-algorithm based methods. Among the BI-based local search methods, Yao et al. [9] propose a two-stage method and show that it is the most efficient local search in terms of solution quality by comparing with the iterative assignment methods $[9,10,21]$ and pair-wise exchange methods $[9,22]$. The iterative assignment methods first assign either the least or the most reliable component to all positions and then iteratively assign the available components to the positions according to their ranks of the BI values until all components are assigned to the system. The difference among the iterative assignment methods is the orders and rules of assigning the components. The pair-wise exchange methods exchange the assignment of pairs of components to match the BI ordering of positions if the system reliability is improved by the exchange. The difference among the pair-wise exchange methods is how to select pairs of components for exchange. In fact, the two-stage method is a combination of the iterative assignment 
method and the pair-wise exchange method. In the two-stage method, the first stage uses the iterative assignment methods to generate the initial solutions; the second stage uses the pair-wise exchange method on these initial solutions with the intention to further improve the solutions. Section 3.1 reviews this BIbased two-stage method in details.

The genetic-algorithm based methods have shown great potential to break local optimum and obtain better solutions than the local search methods [23] and have been used in solving reliability related problems [24]. Yao et al. [6] propose a BI-based genetic local search (BIGLS) algorithm, which embeds a BI-based three-way exchange local research, for addressing the SCAP. The BIGLS outperforms the BIbased two-stage local search in terms of solution quality but scarifies computation time. The BIGLS outperforms the genetic algorithm without the local research, demonstrating the effectiveness of the BIbased local search in combination with genetic algorithm. Cai et al. [25] use a variant of the BIGLS for the $\mathrm{Lin} / \mathrm{Con} / \mathrm{k} / n$ systems, which uses the BI-based two-stage local search rather than the BI-based threeway exchange local search. A $\mathrm{Lin} / \mathrm{Con} / \mathrm{k} / n: \mathrm{F}(\mathrm{G})$ system is an ordered sequence of $n$ components arranged in a line such that the system fails (works) if and only if at least $k$ consecutive components fail (work) [2].

As reviewed above, the BI-based two-stage local search method and the BIGLS are the most efficient heuristics for addressing the SCAP. In this paper, we extend these heuristics to address the MCAP, focusing on embedding of the BI in heuristics and the method of dealing with more than one type of components. The difficulty lies on the interactions of the assignment of various types of components. Then, we investigate the performance of various methods by extensive numerical experiments for the MCAP in the $\operatorname{Lin} / \operatorname{Con} / k / n: F(G)$ systems with multiple-type components and four cases of component reliability. Finally, we propose the efficient BI-based heuristics for addressing the MCAP.

The remainder of the paper is structured as follows. Section 2 establishes a nonlinear binary programming model of the MCAP and shows the numerical examples. Section 3 proposes two methods to deal with multiple types of components: sequential manner and parallel manner, and then designs the BIbased two-stage method for the MCAP (BITSM). Section 4 designs four BI-based genetic local search algorithms. Section 5 evaluates the BITSM, a genetic algorithm without local search, and four BI-based genetic local search algorithms by the numerical experiments. Section 6 gives conclusions and future research.

\section{A nonlinear binary programming model and numerical examples}

To define an MCAP, assume that there are $M$ types of components (positions), $M \geq 1$, and that all components (positions) are independent. When $M=1$, the MCAP becomes the SCAP. Let $n_{\alpha}$ be the number of type $\alpha$ components for $\alpha=1,2, \ldots, M$ and $n$ be the total number of components. Then, $\sum_{\alpha=1}^{M} n_{\alpha}=n$. Define $C_{\alpha}$ as a set of components of type $\alpha$ and $P_{\alpha}$ as a set of positions that can accept type $\alpha$ components for $\alpha=1,2, \ldots, M$. A component or a position is of only one type; that is, $C_{\alpha} \cap C_{\beta}=\Phi$ and $P_{\alpha} \cap P_{\beta}=\Phi$ for $\alpha, \beta=1,2, \mathrm{~K}, M$ and $\alpha \neq \beta$. In the MCAP, a component in $C_{\alpha}$ can be assigned to only one position in $P_{\alpha}$, and a position in $P_{\alpha}$ can accept only one component in $C_{\alpha}$; that is, $\left|C_{\alpha}\right|=\left|P_{\alpha}\right|=n_{\alpha}$ for $\alpha=1,2, \ldots, M$. If $n_{\alpha}=1$, then the component will be assigned to only one position for certain. Therefore, we assume that $n_{\alpha} \geq 2$.

Without loss of generality, assume that the components are indexed according to the order of their types. That is, type $\alpha$ components are in set

$$
C_{\alpha}=\left\{\sum_{\beta=0}^{\alpha-1} n_{\beta}+1, \sum_{\beta=0}^{\alpha-1} n_{\beta}+2, \mathbf{K}, \sum_{\beta=1}^{\alpha} n_{\beta}\right\}
$$

for $\alpha=1,2, \ldots, M$, where $n_{0}=0$. Further, the components of the same type are numbered in nondecreasing order of their reliability; that is, $r_{i} \leq r_{i^{\prime}}$ for $i, i^{\prime} \in C_{\alpha}$ and $i<i^{\prime}$, where $r_{i}$ denotes the reliability of component $i$. The positions are numbered from 1 to $n$ without duplication. Section 2.2 illustrates the notation through an example. 


\subsection{A nonlinear binary programming model}

The MCAP is to find the optimal assignment of components in $C_{\alpha}$ to positions in $P_{\alpha}$ for all $\alpha$ with the objective of maximizing system reliability. Given a set of $n$ components, different component assignment can result in different system reliability.

Define $x_{i j}$ as binary indicator variable, $i \in C_{\alpha}$ and $j \in P_{\alpha}$ for $\alpha=1,2, \ldots, M . x_{i j}=1$ if component $i$ is assigned to position $j$; 0 otherwise. We establish a nonlinear binary programming model of the MCAP as

$$
\begin{array}{lll}
\max & R\left(p_{1}, p_{2}, \mathbf{K}, p_{n}\right) & \\
\text { s.t. } & p_{j}=\sum_{i \in C_{\alpha}} x_{i j} r_{i} & j \in P_{\alpha} ; \alpha=1,2, \mathbf{K}, M \\
& \sum_{i \in C_{\alpha}} x_{i j}=1 & j \in P_{\alpha} ; \alpha=1,2, \mathbf{K}, M \\
& \sum_{j \in P_{\alpha}} x_{i j}=1 & i \in C_{\alpha} ; \alpha=1,2, \mathbf{K}, M \\
& x_{i j} \in\{0,1\} & i \in C_{\alpha}, j \in P_{\alpha} ; \alpha=1,2, \mathbf{K}, M \\
& 0 \leq p_{j} \leq 1 & j=1,2, \mathbf{K}, n
\end{array}
$$

The objective function (3) represents the system reliability $R\left(p_{1}, p_{2}, \mathbf{K}, p_{n}\right)$, which is nonlinear and depends on the assignment of components, $x_{i j}$. Constraint (4) implies that the reliability of position $j$ equals to the reliability of the component, which is assigned to position $j$ and of the same type as position $j$. Constraint (5) ensures that a position of type $\alpha$ can accept only one component of the same type, and constraint (6) ensures that a component of type $\alpha$ can be assigned to only one position of the same type. Constraint (7) defines $x_{i j}$ as binary variables, and constraint (8) specifies the range of $p_{j}$.

\subsection{An illustrative example}

The $\operatorname{Lin} / \mathrm{Con} / \mathrm{k} / n: \mathrm{F}(\mathrm{G})$ systems are important and have many applications such as in networks and telecommunications. For example, consider wireless fidelity (WiFi) or Google Project Fi, which use the strongest signal among the detected signals from different Carriers such as Verizon, AT\&T and T-mobile. To simplify the situation, suppose that there are three Carriers (i.e., three networks of signals) in the commonly covered area and each Carrier operates many relay stations. As sketchily shown in Fig. 1, each relay station can receive and send signal over a certain region (a block), and any area is covered by three consecutive, interlacing relay stations operated by three Carriers. Then, such a signal covering system can be modeled as a $\operatorname{Lin} / \operatorname{Con} / 3 / n: \mathrm{F}$ system with three types of relay stations. The relay stations operated by a Carrier can be exchanged and thus are defined to be one type of components. Apparently, the relay stations operated by different Carriers cannot be exchanged. For a particular area, there is no signal if and only if three consecutive relay stations are failed.

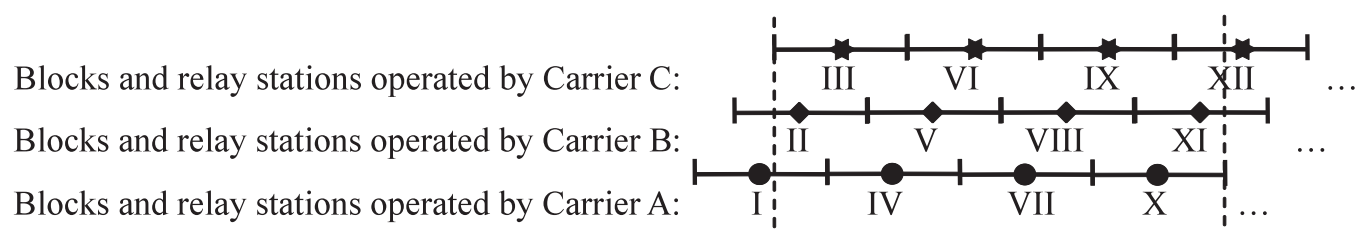

Fig. 1. $\operatorname{Lin} / \mathrm{Con} / 3 / n: \mathrm{F}$ system with three types of components

For illustrative purpose, we consider the area between the dashed lines in Fig. 1, which is commonly covered by twelve consecutive relay stations operated by three Carriers with each Carrier in charge of four relay stations. Then, the reliability of signal covering over the area can be modeled as the Lin/Con/3/12:F system with three types of components and each type of four components. Suppose that 
four relay stations operated by Carrier A have reliability of $0.799,0.838,0.845$, and 0.884 ; thus, these four relay stations are numbered as $1,2,3$, and 4 such that $r_{1}<r_{2}<r_{3}<r_{4}$. Similarly, the four relay stations operated by Carrier $B$ are numbered such as their reliability satisfying $r_{5}=0.709<r_{6}=0.859<r_{7}=0.867<r_{8}=0.871$, and the four relay stations operated by Carrier $\mathrm{C}$ are $r_{9}=0.703<r_{10}=0.781<r_{11}=0.813<r_{12}=0.857$. In this instance, $M=3, n_{1}=n_{2}=n_{3}=4$, and $n=12$; component sets $C_{1}=\{1,2,3,4\}, C_{2}=\{5,6,7,8\}$ and $C_{3}=\{9,10,11,12\}$; position sets $P_{1}=\{\mathrm{I}, \mathrm{IV}, \mathrm{VII}, \mathrm{X}\}$, $P_{2}=\{\mathrm{II}, \mathrm{V}, \mathrm{VIII}, \mathrm{XI}\}$ and $P_{3}=\{\mathrm{III}, \mathrm{VI}, \mathrm{IX}, \mathrm{XII}\}$.

\subsection{Set of numerical examples}

In this paper, we evaluate various heuristics using the numerical experiments on the $\operatorname{Lin} / \mathrm{Con} / \mathrm{k} / n: \mathrm{F}(\mathrm{G})$ systems with $M=k$ types of components. Each type of components has $n_{\alpha}=n / k$ components, assuming that $n$ is divisible by $k$. The $k$ types of positions are arranged sequentially and alternatively, as shown in Fig. 2. In Fig. 2, the positions are indexed from 1 to $n$, and various shapes denote the $k$ types of components. Fig. 1 shows such a $\operatorname{Lin} / \mathrm{Con} / \mathrm{k} / n: \mathrm{F}$ system with $M=k=3$. The reliability of the $\mathrm{Lin} / \mathrm{Con} / \mathrm{k} / n: \mathrm{F}(\mathrm{G})$ systems can be calculated according to $[26,27]$.

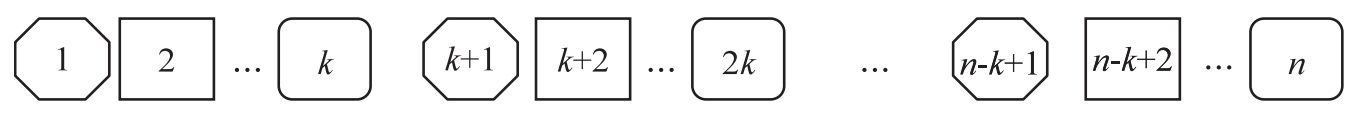

Fig. 2. $\operatorname{Lin} / \operatorname{Con} / k / n: F(G)$ system with multi-type components

We test nine $\operatorname{Lin} / \mathrm{Con} / \mathrm{k} / n: \mathrm{F}$ systems and nine $\operatorname{Lin} / \mathrm{Con} / \mathrm{k} / n: \mathrm{G}$ systems with $k=4,5,10$ and $n=20,40$, 60. For each system, we examine the following four cases on the range of component reliability:

Low reliability of all components is randomly generated from uniform distribution on [0.70, 0.90]

High reliability of all components is randomly generated from uniform distribution on [0.90, 0.99]

Arbitrary reliability of all components is randomly generated from uniform distribution on $[0.70,0.99]$

Alternate reliability of type $\alpha$ components is randomly generated from uniform distributions on $[0.70,0.90]$ and on $[0.90,0.99]$, respectively, for odd $\alpha$ and even $\alpha$.

Given a system and a range of component reliability, we construct 50 instances to avoid the biased results, each instance having a set of randomly generated component reliability. We refer to a set of 50 instances on a system with a range of component reliability as a trial. In our numerical experiments, there are 18 systems, four ranges of component reliability, and thus 72 trials, and there are 50 instances per trial and thus 3600 instances in total. Sections 3, 4, and 5 use these instances for numerical testing.

We program all heuristics in MATLAB R2016a and solve the instances on a compute with Intel ${ }^{\circledR}$ Core $^{\mathrm{TM}}$ i7-6700 $3.40 \mathrm{GHz}$ processors and 16 GB RAM.

\section{The BI-based two-stage method for the MCAP (BITSM)}

As the BI-based two-stage method is the most efficient local search method for the SCAP, we extend it to address the MCAP. We propose two methods to deal with multiple types of components: sequential manner and parallel manner. Section 3.1 reviews the BI-based two-stage method for the SCAP. Section 3.2 presents the sequential and parallel types of algorithms in the two stages for the MCAP along with illustrative examples. Section 3.3 gives the numerical testing to determine the BITSM, and Section 3.4 presents the BITSM.

Let $\boldsymbol{\pi}=\left(\pi_{1}, \pi_{2}, \mathbf{K}, \pi_{n}\right)$ denote an assignment of $n$ components to $n$ positions with $\pi_{i}$ representing the position of component $i$.

3.1. Review of the BI-based two-stage method for the SCAP 
Following the same rule for numbering the components in the MCAP, $n$ components in the SCAP are indexed according to the non-decreasing order of their reliability, i.e., $r_{1} \leq r_{2} \leq \mathbf{K} \leq r_{n}$. Based on [9], the BI-based two-stage method for the SCAP is presented below, in which the ties of comparisons are broken arbitrarily.

\section{The BI-based two-stage method for the SCAP}

Stage A. Iterative assignment to generate two initial solutions $\boldsymbol{\pi}_{1}$ and $\boldsymbol{\pi}_{2}$.

Step A.1. Initially, assign component $1(n)$ to all $n$ positions and set position set $S=\{1,2, \mathbf{K}, n\}$.

Step A.2. Do loop, for $k=1$ to $n-1$

A.2.1 Compute the BI for all positions in $S$ according to Eq. (1).

A.2.2 Find position $j$ in $S$ with the largest (smallest) BI value; assign component $n-k+1$

$(k)$ to position $j$; and remove position $j$ from $S$.

The generated assignment is $\boldsymbol{\pi}_{1}\left(\boldsymbol{\pi}_{2}\right)$.

Stage B. Pair-wise exchange to improve the initial solutions $\boldsymbol{\pi}_{1}$ and $\boldsymbol{\pi}_{2}$.

Step B.1. For an initial assignment $\boldsymbol{\pi}$ ( $\boldsymbol{\pi}_{1}$ and $\boldsymbol{\pi}_{2}$, one at a time), compute the BI for all $n$ positions according to Eq. (1).

Step B.2. Do loop, for $k=n$ to 2

B.2.1 Find position $j$ with the largest BI value among the positions whose current reliability is less than the reliability of position $\pi_{k}$, i.e., component $k$.

B.2.2 If the BI of position $\pi_{k}$ is smaller than the BI of position $j$ and the exchange of the assignment of component $k$ and the component in position $j$ can improve the system reliability, make the exchange and update the BI values of positions; otherwise abandon the exchange.

Step B.3. If there is any exchange in Step B.2, go back to Step B.2; otherwise, stop and return the current assignment.

Stage A generates two initial solutions $\boldsymbol{\pi}_{1}$ and $\boldsymbol{\pi}_{2} . \boldsymbol{\pi}_{1}\left(\boldsymbol{\pi}_{2}\right)$ is generated by first assigning the least (most) reliable component to all the positions, and then iteratively assigns the available component of the highest (lowest) reliability to the position with the largest (smallest) BI until all components are assigned to the system. Stage B further improves the two initial assignments $\boldsymbol{\pi}_{1}$ and $\boldsymbol{\pi}_{2}$ separately, and the final solution is the better one of the two assignments generated in Stage B. Note that when the reliability of all the components is lower than 0.2, the second stage used in [9] is slightly different. Because the component reliability are normally much higher than 0.2 , we use the procedure above, which is used in [9] for general case of component reliability.

\subsection{Sequential and parallel manners of addressing multiple types of components}

To design the BITSM, there are two alternative methods of addressing multiple types of components: sequential manner and parallel manner. The sequential manner is to apply the method for the SCAP to complete the assignment of one type of components and then move to another type in sequence. Alternatively, the parallel manner is to make the operations for each type of components in parallel that improves the assignment for all the types of components simultaneously. With respect to the algorithms in Stage A and Stage B in Section 3.1, we discuss their sequential and parallel extensions for the MCAP below.

\subsubsection{The iterative assignment method in Stage A}

The iterative assignment method in Stage A for the SCAP is extended as the sequential iterative assignment algorithm (SIA) and the parallel iterative assignment algorithm (PIA) for the MCAP.

The sequential iterative assignment in Stage A (SIA) 
Step A.1. Assign all positions in $P_{\alpha}$ with component $\sum_{\beta=0}^{\alpha-1} n_{\beta}+1\left(\sum_{\beta=1}^{\alpha} n_{\beta}\right)$ and set position set $S_{\alpha}=P_{\alpha}$ for $\alpha=1,2, \ldots, M$.

Step A.2. Do loop, for $\alpha=1$ to $M$

Step A.2.1. Do loop, for $k=1$ to $n_{\alpha}-1$

A2.1.1 Compute the BI values of all positions in $S_{\alpha}$ according to Eq. (1).

A2.1.2 Find position $j$ in $S_{\alpha}$ with the largest (smallest) BI value; assign component $\sum_{\beta=1}^{\alpha} n_{\beta}-k+1$ (

$\left.\sum_{\beta=0}^{\alpha-1} n_{\beta}+k\right)$ to position $j$; and remove position $j$ from $S_{\alpha}$.

The generated assignment is $\boldsymbol{\pi}_{1}\left(\boldsymbol{\pi}_{2}\right)$.

\section{The parallel iterative assignment in Stage A (PIA)}

Step A.1. Assign all positions in $P_{\alpha}$ with component $\sum_{\beta=0}^{\alpha-1} n_{\beta}+1\left(\sum_{\beta=1}^{\alpha} n_{\beta}\right)$ and set position set $S_{\alpha}=P_{\alpha}$ for $\alpha=1,2, \ldots, M$.

Step A.2. Do loop, for $k=1$ to $\max _{\alpha=1}^{M}\left\{n_{\alpha}\right\}-1$

Step A.2.1. Compute the BI values of all positions in $S_{\alpha}$ for $\alpha=1,2 \ldots, M$ according to Eq. (1).

Step A.2.2. Do loop, for $\alpha=1$ to $M$

A2.2.1 If $\sum_{\beta=0}^{\alpha-1} n_{\beta}+k<\sum_{\beta=1}^{\alpha} n_{\beta}$, find position $j$ in $S_{\alpha}$ with the largest (smallest) BI value; assign component $\sum_{\beta=1}^{\alpha} n_{\beta}-k+1\left(\sum_{\beta=0}^{\alpha-1} n_{\beta}+k\right)$ to position $j$; and remove position $j$ from $S_{\alpha}$.

The generated assignment is $\boldsymbol{\pi}_{1}\left(\boldsymbol{\pi}_{2}\right)$.

In the loop of SIA, after updating the BI values of positions in $S_{\alpha}$, the assignment is made for only one component of type $\alpha$ among all the components, and the procedure moves to type $\alpha+1$ when all the components of type $\alpha$ are assigned. In the loop of PIA, after updating the BI values of positions in $S_{\alpha}$ for all $\alpha=1,2, \ldots, M$, the assignment is made for one component of each type (i.e., at most $M$ components) if there is any available component.

Example 1 (The SIA and PIA) Consider the illustrative instance in Section 2.2. Using the SIA to generate assignment $\pi_{2}$, Step A.1 assigns components 4,8 and 12 to positions in $P_{1}=\{\mathrm{I}, \mathrm{IV}, \mathrm{VII}, \mathrm{X}\}$, $P_{2}=\{\mathrm{II}, \mathrm{V}, \mathrm{VIII}, \mathrm{XI}\}$ and $P_{3}=\{\mathrm{III}, \mathrm{VI}, \mathrm{IX}, \mathrm{XII}\}$, respectively. That is, assign the most reliable component of each type to all positions of the same type. Then, Step A.2 makes assignment for each type of components in sequence. For $\alpha=1, B I(\mathrm{I})=0.0161, B I(\mathrm{IV})=0.0457, B I(\mathrm{VII})=0.0458$, and $B I(\mathrm{X})=0.0477$, and thus component 1 is assigned to position I, i.e., the type 1 position with the smallest BI value. Then, update the $\mathrm{BI}$ values as $B I(\mathrm{IV})=0.0441, B I(\mathrm{VII})=0.0457$, and $B I(\mathrm{X})=0.0477$, and component 2 is assigned to position IV. Finally, update the BI values as $B I(\mathrm{VII})=0.0448$ and $B I(\mathrm{X})=0.0476$, and component 3 is assigned to position VII. The assignment for type 1 components is done. Similarly, for $\alpha=2$, components 5,6 , and 7 are assigned to positions XI, II, and VIII, respectively, based on the BI values of type 2 positions. For $\alpha=3$, components 9,10 , and 11 are assigned to positions XII, VI, and IX, respectively, based on the BI values of type 3 positions. Therefore, the assignment generated by this SIA is $\pi_{2}=$ (I,IV,VII,X,XI,II,VIII,V,XII,VI,IX,III) with system reliability 0.9605.

Using the PIA to generate $\pi_{2}$, Step A.1 of the PIA is the same as Step A.1 of the SIA. Step A.2 of the PIA assigns one component of each type to a position based on the updated BI values. For $k=1$, the BI values of the twelve positions are $0.0161,0.0288,0.0387,0.0457,0.0409,0.0367,0.0458,0.0409$, $0.0367,0.0477,0.0284$, and 0.0127 . Based on these values, component 1 (type 1 ) is assigned to position I, component 5 (type 2) is assigned to position XI, and component 9 (type 3) is assigned to position XII. Similarly, for $k=2$, after updating the BI values based on the current assignment, components 2 (type 1), 6 (type 2), and 10 (type 3) are assigned to positions IV, VIII, and VI, respectively. For $k=3$, after 
updating the BI values based on the assignment generated at iteration $k=2$, components 3 (type 1), 7 (type 2), and 11 (type 3) are assigned to positions VII, II, and IX, respectively. Therefore, the assignment generated by this PIA is $\pi_{2}=(\mathrm{I}, \mathrm{IV}, \mathrm{VII}, \mathrm{X}, \mathrm{XI}, \mathrm{VIII}, \mathrm{II}, \mathrm{V}, \mathrm{XII}, \mathrm{VI}, \mathrm{IX}, \mathrm{III})$ with system reliability 0.9604 .

\subsubsection{The pair-wise exchange method in Stage B}

Similarly, there are two alternative methods to make the extension of the pair-wise exchange in Stage $\mathrm{B}$ : the sequential pair-wise exchange (SPE) and the parallel pair-wise exchange (PPE), as detailed below.

\section{The sequential pair-wise exchange in Stage B (SPE)}

Step B.1. For an initial assignment $\boldsymbol{\pi}$, do loop for $\alpha=1$ to $M$

Step B.1.1. Compute the BI of all positions in $P_{\alpha}$ according to Eq. (1).

Step B.1.2. Do loop, for $k=\sum_{\beta=1}^{\alpha} n_{\beta}$ to $\sum_{\beta=0}^{\alpha-1} n_{\beta}+2$

B1.2.1 Find position $j$ with the largest BI value among the positions in $P_{\alpha}$ whose current reliability is less than the reliability of position $\pi_{k}$, i.e., component $k$.

B1.2.2 If the $\mathrm{BI}$ of position $\pi_{k}$ is smaller than the BI of position $j$ and the exchange of the assignment of component $k$ and the component in position $j$ can improve the system reliability, make the exchange and update the $\mathrm{BI}$ values of positions in $P_{\alpha}$; otherwise abandon the exchange.

Step B.1.3. If there is any exchange in Step B.1.2, go back to Step B.1.2.

Step B.2. Return the current assignment.

\section{The parallel pair-wise exchange in Stage B (PPE)}

Step B.1. For an initial assignment $\boldsymbol{\pi}$, do loop for $k=1$ to $\max _{\alpha=1}^{M}\left\{n_{\alpha}\right\}-1$

Step B.1.1. Compute the BI values of positions in $P_{\alpha}$ for $\alpha=1,2, \ldots, M$ according to Eq. (1).

Step B.1.2. Do loop, for $\alpha=1$ to $M$

B1.2.1 Let $h=\sum_{\beta=1}^{\alpha} n_{\beta}-k+1$. If $h>\sum_{\beta=0}^{\alpha-1} n_{\beta}+1$, go to Step B1.2.2.

B1.2.2 Find position $j$ with the largest BI value among the positions in $P_{\alpha}$ whose current reliability is less than the reliability of position $\pi_{h}$, i.e., component $h$.

B1.2.3 If the $\mathrm{BI}$ of position $\pi_{h}$ is smaller than the $\mathrm{BI}$ of position $j$ and the exchange of the assignment of component $h$ and the component in position $j$ can improve the system reliability, make the exchange; otherwise abandon the exchange.

Step B.2. If there is any exchange in Step B.1, go back to Step B.1; otherwise, stop and return the current assignment.

Example 2 (The SPE and PPE) For the SPE and PPE to solve the instance in Example 1, we use the assignment generated by the PIA, i.e., $\boldsymbol{\pi}=$ (I,IV,VII,X,XI,VIII,II,V,XII,VI,IX,III), as the initial assignment.

Consider the SPE. First deal with $\alpha=1$ in Step B.1, i.e., type 1 components. For type 1 positions in $P_{1}=\{\mathrm{I}, \mathrm{IV}, \mathrm{VII}, \mathrm{X}\}$, calculate $B I(\mathrm{I})=0.0155, B I(\mathrm{IV})=0.0500, B I(\mathrm{VII})=0.0672$, and $B I(\mathrm{X})=0.1368$ according to Step B.1.1. In Step B.1.2, for $k=4, \pi_{4}=\mathrm{X}$ and position $j=$ VII has the largest BI value among positions I, IV, and VII whose current reliability is less than the reliability of position X, i.e., component 4. Because $B I(\mathrm{VII})=0.0672<B I(\mathrm{X})=0.1368$ and $p_{\mathrm{VII}}=r_{3}<p_{\mathrm{X}}=r_{4}$, no exchange is made for the assignment on positions VII and X. Step B.1.2 continues to $k=3$ and 2, and it turns out that no exchange is made. Because no exchange is made for type 1 components, the assignment for type 1 components is done according to Step B.1.3. 
Then, the SPE moves to $\alpha=2$ in Step B.1. For type 2 positions in $P_{2}=\{\mathrm{II}, \mathrm{V}, \mathrm{VIII}, \mathrm{XI}\}$, calculate $B I$ (II) $=0.0431, B I(\mathrm{~V})=0.0728, B I(\mathrm{VIII})=0.0635$, and $B I(\mathrm{XI})=0.0458$ according to Step B.1.1. In Step B.1.2, for $k=8$, position $\pi_{8}=\mathrm{V}$ and position $j=\mathrm{VIII}$ are evaluated but no exchange is made. For $k=7, \pi_{7}=\mathrm{II}$ and position $j=\mathrm{VIII}$ has the largest $\mathrm{BI}$ value among positions VIII and XI whose current reliability is less than the reliability of position II, i.e., component 7. Because $B I(\mathrm{VIII})=0.0635>B I(\mathrm{II})=0.0431$ and $p_{\mathrm{VIII}}=r_{6}<p_{\mathrm{II}}=r_{7}$, exchanging the assignment on positions II and VIII (i.e., components 7 and 6) is evaluated. Further, this exchange can improve the system reliability; thus, exchange the assignment of components 7 and 6 . The updated assignment is (I,IV,VII,X,XI,II,VIII, V,XII,VI,IX,III) with system reliability 0.9605. Based on this updated assignment, recalculate $B I(\mathrm{II})=0.0431, B I(\mathrm{~V})=0.0729$, $B I(\mathrm{VIII})=0.0634$, and $B I(\mathrm{XI})=0.0459$. Step B.1.2 continues to $k=6$, in which the assignment of components 6 and 5 is exchanged. The updated assignment is (I,IV,VII,X,II,XI,VIII,V,XII,VI,IX,III) with system reliability 0.9609 . Because some exchanges are made, according to Step B.1.3, the next iteration of Step B.1.2 starts with $k=8$ and continues to $k=7$ and 6. No exchange is made in this iteration. Thus, the assignment for type 2 components is done.

Finally, the SPE moves to $\alpha=3$ in Step B. 1 to deal with type 3 components. It turns out that only the assignment of components 11 and 10 is exchanged $(k=11)$. The updated assignment is (I,IV,VII,X,II,XI,VIII,V,XII,IX,VI,III) with system reliability 0.9612. The next iteration of Step B.1.2 for $\alpha=3$ does not make any exchange, and thus the assignment for type 3 components is done. Therefore, the assignment generated by the SPE is (I,IV,VII,X,II,XI,VIII,V,XII,IX,VI,III) with system reliability 0.9612.

Now consider the PPE. For $k=1$, calculate the BI values for all the twelve positions as in Step B.1.1: $0.0155,0.0431,0.0561,0.0500,0.0728,0.0488,0.0672,0.0635,0.0493,0.1368,0.0458$, and 0.0269 . In Step B.1.2, for $\alpha=1, h=4, \pi_{4}=\mathrm{X}$, and position $j=$ VII has the largest BI value among positions I, IV, and VII whose current reliability is less than the reliability of position X, i.e., component 4 . Because $B I(\mathrm{VII})=0.0672<B I(\mathrm{X})=0.1368$ and $p_{\mathrm{VII}}=r_{3}<p_{\mathrm{X}}=r_{4}$, no exchange is made for the assignment on positions $\mathrm{X}$ and VII. Similarly, for $\alpha=2$ and 3, no exchange is made.

The PPE then moves to $k=2$. The BI values for all the twelve positions remain unchanged as for $k=1$ because no exchange of components is made when $k=1$. For $k=2$, no exchange is made for type 1 components $(\alpha=1)$, the assignment of components 7 and 6 is exchanged $(\alpha=2)$, and no exchange is made for type 3 components $(\alpha=3)$. The updated assignment is (I,IV,VII,X,XI,II,VIII, V,XII,VI,IX,III) with system reliability 0.9605 . Then, the PPE moves to $k=3$, and the BI values of all the twelve positions are recalculated based on the updated assignment: $0.0164,0.0431,0.0585,0.0508,0.0729,0.0480$, $0.0647,0.0634,0.0478,0.1359,0.0459$, and 0.0269 . In this step, only the assignment of components 6 and 5 is exchanged $(\alpha=2)$. The updated assignment is (I,IV,VII,X,II,XI,VIII,V,XII,VI,IX,III) with system reliability 0.9609 .

According to Step B.2, because some exchanges are made in the preceding first iteration of Step B.1, the PPE continues to the second iteration of executing Step B.1. For $k=1$, recalculate the BI values of all the positions based on the updated assignment, and no exchange is made. For $k=2$, the assignment of components 3 and $2(\alpha=1)$ and the assignment of components 11 and 10 are exchanged $(\alpha=3)$. The updated assignment is (I, $V I I, I V, X, I I, X I, V I I I, V, X I I, I X, V I, I I I)$ with system reliability 0.9612 . For $k=3$, recalculate the $\mathrm{BI}$ values of all the positions based on the updated assignment, and no exchange is made.

Because some exchanges are made in the second iteration of Step B.1, the PPE continues to the third iteration of Step B.1. For $k=1$, recalculate the BI values of all the positions based on the updated assignment, and only the assignment of components 8 and 7 is exchanged $(\alpha=2)$. For $k=2$ and 3 , no exchange is made. The updated assignment is (I,VII,IV,X,II,XI,V,VIII,XII,IX,VI,III) with system reliability 0.9613 . Because one exchange is made in the third iteration of Step B.1, the PPE continues to the fourth iteration of Step B.1. In the fourth iteration, no exchange is made, and thus the algorithm stops. Therefore, the assignment generated by the PPE is (I,VII,IV,X,II,XI,V,VIII,XII,IX,VI,III) with system reliability 0.9613 , which is, in fact, the optimal solution.

\subsubsection{The three-way exchange method in Stage B}


Except the BI-based pair-wise exchange method, the BI-based three-way exchange method in [6] can also be adapted in Stage B. We extend this local search for the MCAP in both sequential manner and parallel manner, as shown below.

\section{The sequential three-way exchange for the MCAP (STE)}

Step B.1. For an initial assignment $\boldsymbol{\pi}$, do loop for $\alpha=1$ to $M$

Step B.1.1. Compute the BI of all positions in $P_{\alpha}$ according to Eq. (1)

Step B.1.2. Do loop, for $k=1$ to $\left\lceil n_{\alpha} / 2\right\rceil-1$

B1.2.1 Find position $j$ with the largest BI value among the positions in $P_{\alpha}$ whose current reliability is less than the reliability of position $\pi_{h}$ with $h=\sum_{\beta=1}^{\alpha} n_{\beta}-2 k+1$.

B1.2.2 If the relation $B I\left(\pi_{h+1}\right) \geq B I\left(\pi_{h}\right) \geq B I(j)$ is not satisfied, rearrange the three components in positions $\pi_{h+1}, \pi_{h}$ and $j$ such that the reliability values of the components in these three positions are in non-increasing order. If the rearrangement can improve the system reliability, make the rearrangement and update the BI values of positions in $P_{\alpha}$, otherwise abandon the rearrangement.

Step B.1.3. If there is any exchange in Step B.1.2, go back to Step B.1.2.

Step B.2. Return the current assignment.

For each type of components, the inner loop of STE (Step B.1.2) starts with the positions of the most two reliable components and another position with the largest BI value and then makes the exchanges such that the BI values and reliability values of the three positions are in the same order. The exchanges are kept only if they can improve the system reliability. The procedure is processed to the next two reliable components until all the components are addressed. The outer loop (Step B.1) stops when there is no more exchange. When $\alpha=1$ and $n_{1}=n$, the STE becomes the three-way exchange for the SCAP in [6] except that the version in [6] does not have Step B.1.3.

The three-way exchange for the SCAP can be alternatively extended to address the MCAP in parallel manner. The parallel three-way exchange for the MCAP (PTE) is to simultaneously execute the three-way exchange for one group of three components for each type of components, and after updating the BI values, execute the next loop for other groups of three components for all the types of components. The PTE can be easily detailed following the PPE and STE; thus, we do not present the pseudo-code for the PTE to save space.

Example 3 (The STE and PTE) To solve the instance in Example 1, we use the assignment generated by the PIA, i.e., $\pi=(I, I V, V I I, X, X I, V I I I, I I, V, X I I, V I, I X, I I I)$, as the initial assignment in the STE and PTE.

Consider the STE. For $\alpha=1$ in Step B.1, i.e., type 1 positions in $P_{1}=\{\mathrm{I}, \mathrm{IV}, \mathrm{VII}, \mathrm{X}\}$, calculate $B I(\mathrm{I})=$ $0.0155, B I(\mathrm{IV})=0.0500, B I(\mathrm{VII})=0.0672$, and $B I(\mathrm{X})=0.1368$ according to Step B.1.1. In Step B.1.2, for $k=1, h=3, \pi_{3}=$ VII , and position $j=$ IV has the largest BI value among positions I and IV whose current reliability is less than the reliability of position VII, i.e., component 3 . Because $B I(\mathrm{IV})<B I(\mathrm{VII})<$ $B I(\mathrm{X})$ and $p_{\mathrm{IV}}=r_{2}<p_{\mathrm{VII}}=r_{3}<p_{\mathrm{X}}=r_{4}$ in the same order, no three-way exchange is made for the assignment on positions IV, VII and X. Because no three-way exchange is made for type 1 components, the assignment for type 1 components is done according to Step B.1.3.

Then, the STE moves to $\alpha=2$ in Step B.1. For type 2 positions in $P_{2}=\{\mathrm{II}, \mathrm{V}, \mathrm{VIII}, \mathrm{XI}\}$, calculate $B I(\mathrm{II})$ $=0.0431, B I(\mathrm{~V})=0.0728, B I(\mathrm{VIII})=0.0635$, and $B I(\mathrm{XI})=0.0458$ according to Step B.1.1. In Step B.1.2, for $k=1, h=7, \pi_{7}=$ II , and position $j=$ VIII has the largest BI value among positions XI and VIII whose current reliability is less than the reliability of position II, i.e., component 7. Because BI(II) $<$ $\mathrm{BI}(\mathrm{VIII})<\mathrm{BI}(\mathrm{V})$ and $p_{\mathrm{VIII}}=r_{6}<p_{\mathrm{II}}=r_{7}<p_{\mathrm{V}}=r_{8}$, exchanging the assignment of components 6 and 7 makes the BI values and reliability values of positions VIII, II and V in the same order and improves the 
system reliability. The updated assignment is (I,IV,VII,X,XI,II,VIII, V,XII, VI,IX,III) with system reliability 0.9605. Because one exchange is made, according to Step B.1.3, the next iteration of Step B.1.2 is executed and does not make any exchange. Thus, the assignment for type 2 components is done.

Finally, the STE moves to $\alpha=3$ in Step B.1 to deal with type 3 components. It turns out that no exchange is made, and thus the assignment for type 3 components is done. Therefore, the assignment generated by the STE is (I,IV,VII,X,XI,II,VIII,V,XII,VI,IX,III) with system reliability 0.9605 .

Now consider the PTE. For $k=1$, calculate the BI values for all the twelve positions: 0.0155, 0.0431, $0.0561,0.0500,0.0728,0.0488,0.0672,0.0635,0.0493,0.1368,0.0458$, and 0.0269 . For $\alpha=1$, $h=\sum_{\beta=1}^{\alpha} n_{\beta}-2 k+1=3, \pi_{3}=\mathrm{VII}$, and position $j=$ IV has the largest BI value among positions I and IV whose current reliability is less than the reliability of position VII, i.e., component 3. Because $B I(\mathrm{IV})=$ $0.0500<B I(\mathrm{VII})=0.0672<B I(\mathrm{X})=0.1368$ and $p_{\mathrm{IV}}=r_{2}<p_{\mathrm{VII}}=r_{3}<p_{\mathrm{X}}=r_{4}$ in the same order, no threeway exchange is made. For $\alpha=2, h=7, \pi_{7}=\mathrm{II}$, and position $j=$ VIII is the only type 2 position whose current reliability is less than the reliability of position II. Because $B I(\mathrm{II})=0.0431<B I(\mathrm{VIII})=0.0635<$ $B I(\mathrm{~V})=0.0728$ and $p_{\mathrm{VIII}}=r_{6}<p_{\mathrm{II}}=r_{7}<p_{\mathrm{V}}=r_{8}$, exchanging the assignment of components 6 and 7 makes the BI values and reliability values of positions II, VIII and V in the same order and improves the system reliability. The updated assignment is (I,IV,VII,X,XI,II,VIII, V,XII, VI,IX,III) with system reliability 0.9605 . For $\alpha=3, h=11, \pi_{11}=\mathrm{IX}$, and position $j=\mathrm{VI}$ is the only type 3 position whose current reliability is less than the reliability of position IX. The BI values and reliability values of positions IX, VI and III are in the same order, and thus no three-way exchange is made. Just like the PPE, the $\mathrm{BI}$ value is not updated for $\alpha=1,2$, and 3 .

The preceding loop is executed for $k$ from 1 to $\max _{\alpha=1}^{3}\left\lceil n_{\alpha} / 2\right\rceil-1$. In this instance, $\max _{\alpha=1}^{3}\left\lceil n_{\alpha} / 2\right\rceil-1=1$. Because one three-way exchange is made in the preceding loop, the next iteration of the loop continues. The BI values of all the twelve positions are recalculated based on the updated assignment: $0.0164,0.0431,0.0585,0.0508,0.0729,0.0480,0.0647,0.0634,0.0478,0.1359,0.0459$, and 0.0269 . The BI values and reliability values of the positions are always in the same order, and, thus, no three-way exchange is made. Therefore, the algorithm stops, and the assignment generated by the PTE is (I,IV,VII,X,XI,II,VIII,V,XII,VI,IX,III) with system reliability 0.9605.

\subsection{Design of the BITSM}

As shown in Section 3.2, there are four alternatives in Stage A (the SIA generating two assignments $\boldsymbol{\pi}_{1}$ and $\boldsymbol{\pi}_{2}$ and the PIA generating two assignments $\boldsymbol{\pi}_{1}$ and $\boldsymbol{\pi}_{2}$ ) and four alternatives in Stage B (the SPE, PPE, STE, and PTE) to construct the two-stage method. Therefore, there are 16 combinations of the two stages; for example, SIA $\left(\boldsymbol{\pi}_{1}\right)$-SPE means that Stage A uses the SIA to generate initial solution $\boldsymbol{\pi}_{1}$ and Stage B uses the SPE to further improve this initial solution. Table 1 lists in its first two rows the algorithms used in Stage A and Stage B for the 16 combinations and a special combination with two initial solutions generated by the PIA in Stage A and the PPE in Stage B. 
Table 1. Comparisons of the algorithms in the two-stage framework

\begin{tabular}{|c|c|c|c|c|c|c|c|c|c|c|c|c|c|c|c|c|c|}
\hline \multirow{2}{*}{\begin{tabular}{r|} 
Stage A \\
Stage $\mathbf{B}$
\end{tabular}} & \multicolumn{4}{|c|}{ 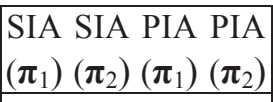 } & \multirow{2}{*}{\multicolumn{4}{|c|}{ 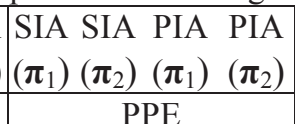 }} & \multicolumn{4}{|c|}{ 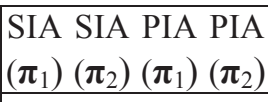 } & \multicolumn{4}{|c|}{  } & \multirow{2}{*}{$\begin{array}{c}\text { PIA } \\
\left(\boldsymbol{\pi}_{1}+\boldsymbol{\pi}_{2}\right) \\
\mathrm{PPE}\end{array}$} \\
\hline & & $\mathrm{SH}$ & $\mathrm{PE}$ & & & & & & & & $\Gamma E$ & & & $\mathrm{P} T$ & & & \\
\hline 4/20:H & 7 & 10 & 18 & 60 & 34 & 33 & 72 & 115 & 8 & 11 & 18 & 60 & 0 & 0 & 3 & 5 & 153 \\
\hline 5/20: & 11 & 11 & 23 & 32 & 46 & 43 & 105 & 120 & 4 & 4 & 18 & 23 & 1 & 1 & 2 & 2 & 168 \\
\hline 10/20: & 126 & 123 & 148 & 147 & 129 & 130 & 163 & 162 & 3 & 4 & 64 & 64 & 3 & 4 & 64 & 64 & 163 \\
\hline 4/40:H & 1 & 1 & 14 & 2 & 17 & 21 & 114 & 38 & 0 & 0 & 13 & 2 & 0 & 0 & 2 & 0 & 152 \\
\hline 5/40: & 0 & 0 & 5 & 4 & 23 & 16 & 95 & 58 & 0 & 0 & 3 & 3 & 0 & 0 & 0 & 0 & 153 \\
\hline 10/40: & 1 & 1 & 3 & 2 & 28 & 22 & 68 & 87 & 0 & 0 & 2 & 1 & 0 & 0 & 0 & 0 & 152 \\
\hline 4/60:H & 0 & 0 & 4 & 0 & 10 & 18 & 150 & 19 & 0 & 0 & 5 & 1 & 0 & 0 & 0 & 0 & 169 \\
\hline 5/60:H & 1 & 0 & 1 & 0 & 18 & 18 & 124 & 39 & 1 & 0 & 0 & 0 & 0 & 0 & 0 & 0 & 163 \\
\hline 10/60:H & 0 & 1 & 0 & 0 & 11 & 25 & 79 & 84 & 0 & 0 & 0 & 0 & 0 & 0 & 0 & 0 & 163 \\
\hline $4 / 20: \mathrm{C}$ & 12 & 71 & 26 & 31 & 20 & 78 & 49 & 42 & 11 & 72 & 25 & 30 & 3 & 30 & 6 & 12 & 89 \\
\hline $0: 0$ & 28 & 75 & 29 & 69 & 42 & 82 & 43 & 83 & 14 & 65 & 24 & 49 & 1 & 38 & 1 & 29 & 118 \\
\hline 10/20: & 111 & 164 & 147 & 181 & 141 & 164 & 150 & 181 & 19 & 136 & 95 & 168 & 19 & 136 & 95 & 168 & 182 \\
\hline $4 / 40: \mathrm{C}$ & 2 & 9 & 23 & 14 & 7 & 28 & 70 & 53 & 2 & 9 & 20 & 13 & 0 & 0 & 0 & 8 & 123 \\
\hline $5 / 40: d$ & 3 & 15 & 7 & 19 & 12 & 56 & 39 & 71 & 1 & 10 & 4 & 14 & 0 & 0 & 0 & 8 & 110 \\
\hline $10 / 40:$ & 3 & 43 & 10 & 53 & 35 & 59 & 59 & 91 & 0 & 32 & 9 & 30 & 0 & 5 & 1 & 9 & 139 \\
\hline $4 / 60: \mathrm{d}$ & 6 & 15 & 24 & 23 & 8 & 28 & 53 & 105 & 9 & 17 & 20 & 20 & 5 & 11 & 18 & 6 & 148 \\
\hline $5 / 60: \mathrm{d}$ & 1 & 6 & 4 & 13 & 0 & 20 & 47 & 109 & 0 & 1 & 3 & 9 & 0 & 0 & 0 & 2 & 156 \\
\hline 10/60: & 0 & 6 & 5 & 29 & 29 & 23 & 33 & 105 & 0 & 5 & 2 & 18 & 0 & 0 & 0 & 3 & 138 \\
\hline Tota & 313 & 551 & 491 & 679 & 610 & 864 & 1513 & 1562 & 72 & 366 & 325 & 505 & 32 & 225 & 192 & 316 & 2639 \\
\hline
\end{tabular}

To compare these alternatives, we use the 17 versions of the two-stage method to solve all the 3600 instances in Section 2.3. For an instance, these algorithms may obtain the different solutions. The first 16 versions of the two-stage method take 0.0002 to 0.0247 seconds for solving an instance, and the version using two initial solutions in the last column in Table 1 takes 0.0005 to 0.039 seconds. That is, these local search methods are very efficient. Thus, we do not report the computation times in details.

Table 1 shows the tallied computational results for the $\operatorname{Lin} / \mathrm{Con} / \mathrm{k} / n: \mathrm{F}$ or $\mathrm{G}$ systems, for which the first column in Table 1 specifies the $k(k=M)$ and $n$ values. Recall from Section 2.3 that there are four cases of component reliability and 50 instances for a system with one case of component reliability; thus, there are 200 instances in total for a system. For a particular system (e.g., Lin/Con/4/20:F system), the associated row (starting with 4/20:F) gives the numbers of instances among the 200 instances, for which the corresponding algorithm obtains the best solution among the solutions generated by the 17 versions of the two-stage method. For example, the algorithm of SIA $\left(\pi_{1}\right)$-SPE obtains the best solutions for seven instances. The last row gives the numbers of instances among the total 3600 instances associated with all the 18 systems, for which the corresponding algorithm achieves the best solutions. Note that different versions of the two-stage method may obtain the same best solution for an instance.

As shown in Table 1, we observe the following main points.

a. Consider the four alternatives in Stage A. For the same algorithm in Stage B, the PIA $\left(\boldsymbol{\pi}_{1}\right)$ always performs better than the $\operatorname{SIA}\left(\boldsymbol{\pi}_{1}\right)$, and the $\operatorname{PIA}\left(\boldsymbol{\pi}_{2}\right)$ performs better than the $\operatorname{SIA}\left(\boldsymbol{\pi}_{2}\right)$. For example, when using the SPE in Stage B, the algorithms of PIA $\left(\boldsymbol{\pi}_{1}\right)-\operatorname{SPE}, \operatorname{SIA}\left(\boldsymbol{\pi}_{1}\right)-\operatorname{SPE}, \operatorname{PIA}\left(\boldsymbol{\pi}_{2}\right)-\operatorname{SPE}$, and $\operatorname{SIA}\left(\boldsymbol{\pi}_{2}\right)$-SPE obtain, in overall, the best solutions for $491,313,679$, and 551 instances, respectively, as shown in the last row. Therefore, the PIA, i.e., the parallel manner, performs better than the SIA, i.e., the sequential manner.

b. Consider the four algorithms in Stage B. For the same algorithm in Stage A, the PPE outperforms the SPE, the SPE outperforms the STE, and the STE outperforms the PTE. For example, using the $\operatorname{PIA}\left(\boldsymbol{\pi}_{2}\right)$ in Stage A, the algorithms of PIA $\left(\boldsymbol{\pi}_{2}\right)$-PPE, PIA $\left(\boldsymbol{\pi}_{2}\right)$-SPE, PIA $\left(\boldsymbol{\pi}_{2}\right)$-STE, and PIA $\left(\boldsymbol{\pi}_{2}\right)$-PTE obtain the best solutions for 1562, 679, 505, and 316 instances, respectively, as shown in the last row. Over the four versions of the two-stage method using the same stage B algorithm, the SPE generates 2034 best solutions (sum of 313, 551, 491, and 679), and similarly the PPE, STE, and 
PTE generate 4549, 1268, and 765 best solutions, respectively. Therefore, the SPE and the PPE (i.e., the pair-wise exchange) dominantly outperform the STE and the PTE (i.e., the three-way exchange), possibly because the exchange of two components is more subtle than the exchanges of three components. Further, the PPE, i.e., the parallel manner, performs better than the SPE, i.e., the sequential manner.

c. Among the first 16 versions of the two-stage method, the algorithms of PIA( $\left.\boldsymbol{\pi}_{2}\right) \operatorname{PPE}$ and $\operatorname{PIA}\left(\boldsymbol{\pi}_{1}\right)$ PPE are the best two, obtaining the best solutions for 1562 and 1513 instances, respectively.

d. Motivated by point (c), if both the PIA $\left(\boldsymbol{\pi}_{1}\right)$ and $\operatorname{PIA}\left(\boldsymbol{\pi}_{2}\right)$ are used in Stage A to generate two initial solutions and the PPE is used in Stage B as the algorithm in the last column, then the algorithm obtains the best solutions for 2639 instances in total.

\subsection{Description of the BITSM}

Based on the observations in Section 3.3, we propose the BITSM below, which uses the PIA ( $\boldsymbol{\pi}_{1}$ and $\pi_{2}$ ) in Stage A and the PPE in Stage B. It is similar to the BI-based two-stage method for the SCAP in Section 3.1, which also uses two initial solutions.

\section{The BI-based two-stage method for the MCAP (BITSM)}

Stage A. Use the PIA to generate two initial solutions $\boldsymbol{\pi}_{1}$ and $\boldsymbol{\pi}_{2}$.

Stage B. Using the initial solutions from Stage A separately, solve the instance by the PPE, and the final solution is the better one of the two assignments generated.

Example 4 (The BITSM) Consider the illustrative instance in Section 2.2 and Examples 1, 2 and 3. In Stage A, the PIA generates one solution $\boldsymbol{\pi}_{1}=$ (I,IV,VII,X,XI,II,VIII,V,XII,VI,IX,III) with system reliability 0.9605 by first assigning the least reliable component of each type to all the positions of the same type. The PIA generates another solution $\boldsymbol{\pi}_{2}=$ (I,IV,VII,X,XI,VIII,II,V,XII,VI,IX,III) with system reliability 0.9604 as in Example 1. In Stage B, using initial solution $\boldsymbol{\pi}_{1}$ or $\boldsymbol{\pi}_{2}$, the PPE generates the same assignment (I,VII,IV,X,II,XI,V,VIII,XII,IX,VI,III) with system reliability 0.9613 as in Example 2. Thus, the final solution generated by the BITSM is assignment (I,VII,IV,X,II,XI,V,VIII,XII,IX,VI,III) with system reliability 0.9613 , which is the optimal assignment.

\section{Design of the BI-based genetic local search algorithms}

Section 4.1 shows the genetic algorithm with local search. Sections $4.2-4.6$ specify the main operators and the local search used in this genetic local search.

\subsection{The genetic local search algorithm}

A generation has $m$ chromosomes, and a chromosome is coded as an assignment $\pi$ with gene $\pi_{i}$ representing the position of component $i$. The genetic algorithm with local search is presented below.

\section{The genetic local search algorithm}

Step 1. The initial generation includes $m-1$ randomly generated chromosomes and one chromosome generated by the BITSM. Perform the local search on each initial chromosome.

Step 2. Compute the fitness for each chromosome according to Eqs. (9) and (10) (see Section 4.2).

Step 3. If the number of generations reaches a pre-specified maximal number of generations or the current generation satisfies termination condition in Eq. (11) (see Section 4.3), then perform the local search on the best chromosome and return the final solution.

Step 4. Perform elitism strategy by selecting $\lceil m \beta\rceil$ elitism chromosomes with the highest fitness values in the current generation, where $\beta$ is the elitist rate, $0 \leq \beta \leq 1$. 
Step 5. Generate $m-\lceil m \beta\rceil$ offspring chromosomes.

Step 5.1. For selected parents, perform the partial matched crossover (PMX) to generate a child chromosome with crossover probability $P_{c}, 0 \leq P_{c} \leq 1$ (see Section 4.4), and choose the first selected parent chromosome as a child chromosome with probability $1-P_{c}$.

Step 5.2. Perform the swap mutation on the child chromosome with mutation probability $P_{m}$, $0 \leq P_{m} \leq 1$ (see Section 4.5).

Step 5.3. Perform the local search on each mutated child chromosome to generate an offspring chromosome. For unmutated child chromosomes, perform the local search on the chromosomes that are better than the best chromosome in the current generation and copy the rest of the chromosomes directly as offspring chromosomes.

Step 6. Use the $\lceil m \beta\rceil$ elitism chromosomes and $m-\lceil m \beta\rceil$ offspring chromosomes to replace the current generation. Then go to Step 2.

\subsection{Fitness scaling}

For chromosome $t=1,2 \ldots m, f_{t}$ is the system reliability corresponding to chromosome $t$, and $F_{t}$ is the scaled fitness value. $F_{t}$ is defined using a linear scaling function with coefficients $\phi_{1}$ and $\phi_{2}$ as [28]

$$
F_{t}=\phi_{1} f_{t}+\phi_{2}
$$

Let $\kappa$ be the scaling factor. The coefficients $\phi_{1}$ and $\phi_{2}$ are determined as

$$
\phi_{1}=\frac{(\kappa-1) \cdot f_{\text {avg }}}{\kappa\left(f_{\text {avg }}-f_{\text {min }}\right)+\left(f_{\max }-f_{\text {avg }}\right)} \text { and } \phi_{2}=\left(1-\phi_{1}\right) \cdot f_{\text {avg }}
$$

where $f_{\max }, f_{\min }$, and $f_{\text {avg }}$ denote the maximum, minimum, and average values of $f_{t}$ 's in one generation, respectively. Let $F_{\max }, F_{\min }$, and $F_{\text {avg }}$ denote the maximum, minimum, and average values of $F_{t}$ 's, respectively. The formulas in Eq. (10) is derived such that $F_{\text {avg }}=f_{\text {avg }}$ and $F_{\max }=\kappa F_{\min }$. The value of scaling factor $\kappa$ should be chosen to balance the premature (large $\kappa$ ) and the divergence (small $\kappa$ ).

\subsection{Termination conditions}

One termination condition is

$$
\frac{\kappa\left(f_{\text {avg }}-f_{\min }\right)+\left(f_{\text {max }}-f_{\text {avg }}\right)}{\kappa\left(f_{\max }-f_{\text {min }}\right)} \geq \varepsilon
$$

where tolerance $\varepsilon$ is less than and close to 1 . Eq. (11) is equivalent to $F_{\text {avg }} / F_{\max } \geq \varepsilon$. The termination condition in [6] and [29] is

$$
\kappa\left(f_{\text {avg }}-f_{\min }\right)+\left(f_{\max }-f_{\text {avg }}\right) \leq \eta
$$

for tolerance $\eta, \eta$ close to 0 . Termination condition in Eq. (11) is superior to condition in Eq. (12) because the value of $\eta$ ought to be determined based on the magnitude of fitness values and thus has to be changed for instances, which is inconvenient in use.

\subsection{Parent selection and crossover}

The selection of parent chromosomes for crossover is based on the biased roulette wheel criterion [29] with probability $F_{t} / \sum_{j=1}^{m} F_{j}$ for $t=1,2, \mathbf{K}, m$. A chromosome can be re-selected as parents.

Under the PMX, two points, picked uniformly at random, define a matching section between two parent chromosomes according to which a crossing of position-by-position exchanges is implemented. The PMX guarantees that the exchanges are made within the same type of components. For example, Fig. 
3 shows two parent chromosomes and two randomly picked crossing points 3 and 6 . Then, according to the sections of genes 3, 4 and 5 in the two parents, exchanging positions V, VII and II with positions I, VII and IV in Parent 1, respectively, produces a Child chromosome (III,V,I,VII,IV,II,VI,VIII).

\begin{tabular}{rlllll|l:lll} 
Parent 1 & III & I & V & VII & II & IV & VI & VIII \\
Parent 2 & V & III & I & VII & IV & VIII & II & VI \\
Child = & III & V & I & VII & IV & II & VI & VIII
\end{tabular}

Fig. 3. The partial matched crossover (PMX)

\subsection{Mutation}

For the mutation, two components are randomly selected within the same type of components, and the corresponding genes, i.e., the assignment of the two components, are swapped. For the MCAP, there are two methods to execute the swap mutation on a chromosome. One method is to execute the swaps of two genes for each type of components, i.e., $M$ times of swaps in total (MS). An alternative method is to execute the swap of two genes for only one randomly selected type of components, i.e., only one swap (OS).

To compare the effectiveness of MS and OS, we revise the genetic local search in Section 4.1 by removing the local search from Steps 1, 3, and 5.3 for simplicity. We use the genetic algorithm with the MS and the genetic algorithm with the OS to solve all the 72 trials in Section 2.3. Referring to [6, 28, 29] and the preliminary study in Section 5.1, set parameters $m=[1.5 n\rceil$ ( $n$ being the number of components), $\beta=0.1, \kappa=1000, P_{c}=0.8, P_{m}=0.05, \varepsilon=0.985$, and the maximal number of generations as 800 .

Table 2 presents the computational results. Columns "MS $>\mid<O$ OS" give two numbers separated by "I" in each cell, which is associated with a trail of 50 instances. Recall that the trial is defined on the system specified by the first column and with a case of component reliability specified by the first row. The first (second) number in a cell is the number of instances, for which the genetic algorithm with the MS obtains strictly better (worse) solution than that with the OS. The number of instances for which the two methods obtain the same solutions equals 50 minus the two numbers in the cell. For example, among the 50 instances in the trial associated with the $\mathrm{Lin} / \mathrm{Con} / 4 / 40: \mathrm{G}$ system and low component reliability, there are 20 instances for which the genetic algorithm with the MS obtains better solutions than that with the OS, 13 instances for which the genetic algorithm with the MS obtains worse solutions than that with the OS, and 17 instances for which the genetic algorithm with the MS and the genetic algorithm with the OS obtain the same solutions. 
Table 2. Comparisons of the MS and the OS

\begin{tabular}{|c|c|c|c|c|c|c|c|c|c|c|c|c|}
\hline \multirow{3}{*}{$\begin{array}{r}\text { comp. } \\
\text { rel. } \\
\text { systems }\end{array}$} & \multicolumn{3}{|c|}{ Low } & \multicolumn{3}{|c|}{ High } & \multicolumn{3}{|c|}{ Alternate } & \multicolumn{3}{|c|}{ Arbitrary } \\
\hline & \multirow[b]{2}{*}{$\mathrm{MS}>\mid<\mathrm{OS}$} & \multicolumn{2}{|c|}{ \# of gens } & \multirow[b]{2}{*}{$\mathrm{MS}>1<\mathrm{OS}$} & \multicolumn{2}{|c|}{ \# of gens } & \multirow[b]{2}{*}{$\mathrm{MS}>\mid<\mathrm{OS}$} & \multicolumn{2}{|c|}{ \# of gens } & \multirow[b]{2}{*}{$\mathrm{MS}>1<\mathrm{OS}$} & \multicolumn{2}{|c|}{ \# of gens } \\
\hline & & OS & MS & & $\mathrm{OS}$ & MS & & OS & MS & & OS & MS \\
\hline $4 / 20: F$ & $0 \backslash 0$ & 17 & 23 & $0 \backslash 2$ & 18 & 28 & $2 \backslash 2$ & 18 & 25 & $4 \backslash 1$ & 19 & 28 \\
\hline $5 / 20: F$ & $2 \backslash 1$ & 18 & 28 & $1 \backslash 1$ & 17 & 27 & $0 \backslash 2$ & 19 & 24 & $3 \backslash 2$ & 20 & 28 \\
\hline 10/20:F & $1 \backslash 0$ & 14 & 25 & $2 \backslash 5$ & 15 & 28 & $2 \backslash 0$ & 14 & 27 & $6 \backslash 2$ & 15 & 31 \\
\hline 4/40:F & $4 \backslash 0$ & 66 & 300 & $10 \backslash 0$ & 79 & 315 & $7 \backslash 1$ & 107 & 350 & $3 \backslash 0$ & 114 & 316 \\
\hline 5/40:F & $7 \backslash 1$ & 72 & 300 & $8 \backslash 2$ & 100 & 310 & $13 \backslash 2$ & 84 & 368 & $14 \backslash 0$ & 87 & 397 \\
\hline 10/40:F & $14 \backslash 3$ & 55 & 375 & $33 \backslash 3$ & 47 & 369 & $26 \backslash 1$ & 45 & 485 & $29 \backslash 6$ & 62 & 578 \\
\hline 4/60:F & $4 \backslash 0$ & 32 & 176 & $11 \backslash 1$ & 27 & 187 & $5 \backslash 0$ & 25 & 163 & $5 \backslash 0$ & 26 & 173 \\
\hline $5 / 60: F$ & $1 \backslash 1$ & 33 & 163 & $5 \backslash 0$ & 27 & 186 & $9 \backslash 2$ & 31 & 213 & $3 \backslash 0$ & 29 & 186 \\
\hline 10/60:F & $13 \backslash 1$ & 30 & 268 & $18 \backslash 2$ & 38 & 248 & $22 \backslash 1$ & 28 & 269 & $14 \backslash 1$ & 33 & 143 \\
\hline $4 / 20: G$ & $12 \backslash 12$ & 19 & 29 & $20 \backslash 20$ & 21 & 33 & $12 \backslash 12$ & 20 & 27 & $10 \backslash 12$ & 21 & 31 \\
\hline $5 / 20: \mathrm{G}$ & $6 \backslash 8$ & 16 & 22 & $11 \backslash 11$ & 19 & 33 & $14 \backslash 8$ & 20 & 32 & $9 \backslash 10$ & 17 & 26 \\
\hline 10/20:G & $2 \backslash 3$ & 12 & 21 & $1 \backslash 2$ & 11 & 24 & $2 \backslash 0$ & 12 & 23 & $2 \backslash 1$ & 12 & 22 \\
\hline $4 / 40: G$ & $20 \backslash 13$ & 126 & 380 & $31 \backslash 17$ & 117 & 375 & $27 \backslash 17$ & 149 & 401 & $30 \backslash 12$ & 151 & 512 \\
\hline $5 / 40: \mathrm{G}$ & $20 \backslash 13$ & 103 & 317 & $34 \backslash 11$ & 153 & 486 & $29 \backslash 8$ & 123 & 445 & $24 \backslash 13$ & 115 & 413 \\
\hline 10/40:G & $6 \backslash 5$ & 49 & 195 & $10 \backslash 5$ & 59 & 240 & $9 \backslash 4$ & 48 & 265 & $7 \backslash 7$ & 47 & 193 \\
\hline 4/60:G & $26 \backslash 15$ & 38 & 145 & $2 \backslash 2$ & 13 & 22 & $36 \backslash 11$ & 43 & 161 & $31 \backslash 14$ & 37 & 131 \\
\hline $5 / 60: \mathrm{G}$ & $22 \backslash 9$ & 28 & 163 & $31 \backslash 16$ & 42 & 179 & $33 \backslash 7$ & 29 & 202 & $34 \backslash 4$ & 33 & 197 \\
\hline $10 / 60: G$ & $10 \backslash 9$ & 28 & 159 & $27 \backslash 5$ & 33 & 223 & $24 \backslash 4$ & 30 & 211 & $19 \backslash 6$ & 33 & 203 \\
\hline
\end{tabular}

If the first number in a cell in columns "MS $>\mid<\mathrm{OS}$ " is larger (smaller) than the second number, then the MS performs better (worse) than the OS for the trail associated with the cell. As shown in columns "MS $>\mid<O S$ ", the MS obtains the better solutions than the OS for more or equal number of instances in 63 out of 72 trails. Thus, the MS performs better than the OS in general.

In Table 2, columns "\# of gens" record the average numbers of generations performed for solving one instance in the corresponding trials. As shown in these columns, the genetic algorithm with the MS takes more generations to converge and terminate than the genetic algorithm with the OS for all the 72 trials because the multiple swaps increase the variation of chromosomes and the diversity of generations more significantly than the one swap. Consequently, the genetic algorithm with the MS takes more time (1.835 seconds per instance on average) than the OS ( 0.280 seconds per instance on average) but is still efficient.

Considering the solution quality as the first priority, the MS is used in the mutation. For example, for the Child chromosome in Fig. 3, suppose that positions III and V of type 1 and positions II and VIII of type 2 are randomly selected. After swapping positions III and V and swapping positions II and VIII, a mutated chromosome (V,III,I,VII,IV,VIII,VI,II) is produced.

\subsection{The BI-based local search algorithms}

Considering the solution quality and computational efficiency, Yao et al. [6] and Lim et al. [28] recommend not performing the local search on every new chromosome for the SCAP and quadratic assignment problems, respectively. Since the MCAP is an extension of the SCAP, the local search is only performed on some promising new chromosomes in Steps 1, 3, and 5.3 in the genetic local search in Section 4.1.

We can use the PPE, SPE, PTE, and STE as the local search in the genetic local search. Based on the numerical testing in Section 3.3, for the SPE and STE, the loop in Step B.1.2 for one type of components is executed 7 times per instance on average. For the PPE and PTE, the average execution times of the loop in Step B.1 are 18 and 11 times per instance, respectively. The local search in the genetic local search can be implemented for hundreds to thousands of times. Thus, in order to balance the computation time and the quality of chromosomes, the PPE, SPE, PTE, and STE in Sections 3.2.2 and 3.2.3 can be revised to execute their main loops only one time for one chromosome when using them in the genetic 
local search. That is, Step B.1.3 is removed from the SPE and STE, and Step B.2 is removed from the PPE and PTE. We refer to the genetic local search in Section 4.1 using the revised PPE, revised SPE, revised PTE, and revised STE as the GLPPE, GLSPE, GLPTE, and GLSTE, respectively. Removing the local search from Steps 1, 3, and 5.3 as in Section 4.5, we have the genetic algorithm for the MCAP and refer to it as the GAM. Section 5 compares the performances of the five genetic algorithms.

\section{Numerical experiments on the BITSM, GAM, GLSPE, GLPPE, GLSTE, and GLPTE}

Section 5.1 conducts a preliminary study to determine the maximal number of generations for the genetic algorithms - the GAM, GLSPE, GLPPE, GLSTE, and GLPTE - to solve the MCAP. Section 5.2 presents the numerical experiments to evaluate the performances of the BITSM, GAM, GLSPE, GLPPE, GLSTE, and GLPTE and to make recommendations on the final BI-based methods.

\subsection{Preliminary study on the genetic algorithms}

To evaluate the convergence and capability of the genetic algorithms for addressing the MCAP, we use the GAM, GLSPE, GLPPE, GLPTE, and GLSTE to solve four instances on the Lin/Con/4/60:F system, each with one case of component reliability: Low, High, Arbitrary, and Alternative as specified in Section 2.3.

Let the single termination condition be the maximal number of generations as 1200. Fig. 4 shows the system reliability of the current best solutions for 1200 generations. The current best solutions from the GAM, GLSPE, GLPPE, GLSTE, and GLPTE remain unchanged after about 800 generations. Thus, the maximal number of generations is set to 800 for the GAM, GLSPE, GLPPE, GLSTE, and GLPTE in Sections 4.5 and 5.2.

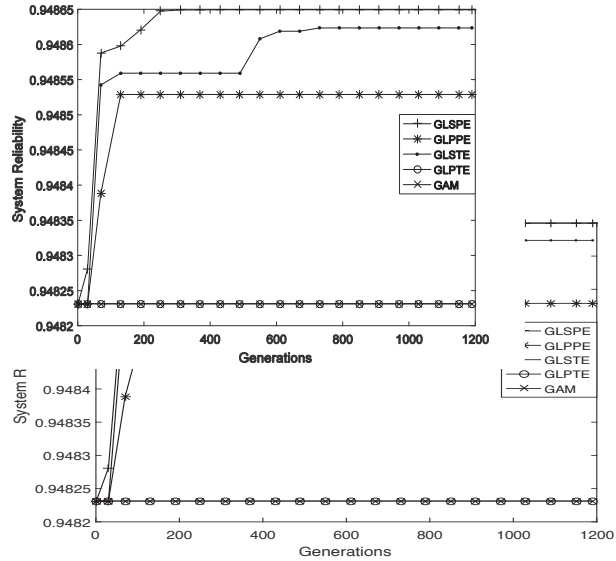

(a). Low component reliability

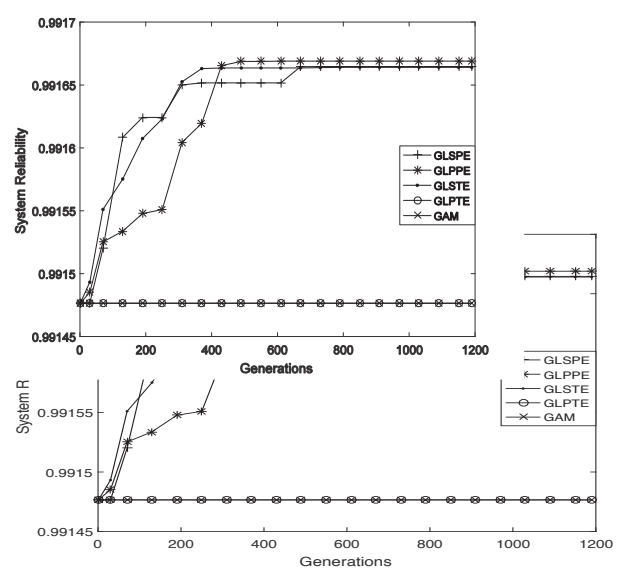

(c). Arbitrary component reliability

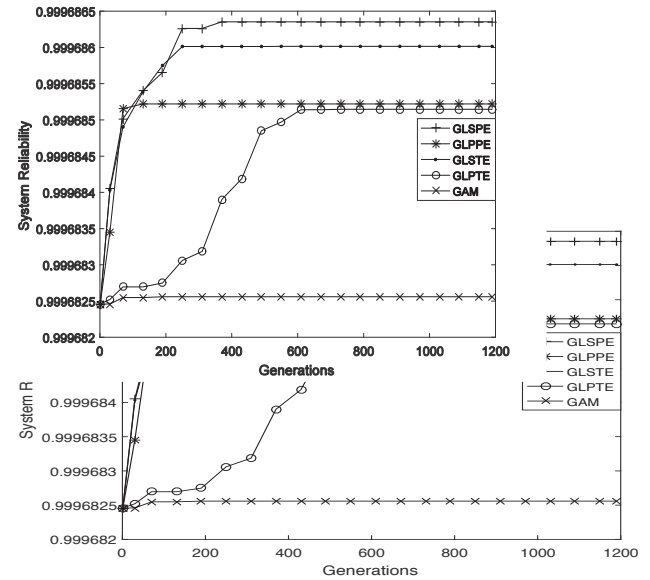

(b). High component reliability

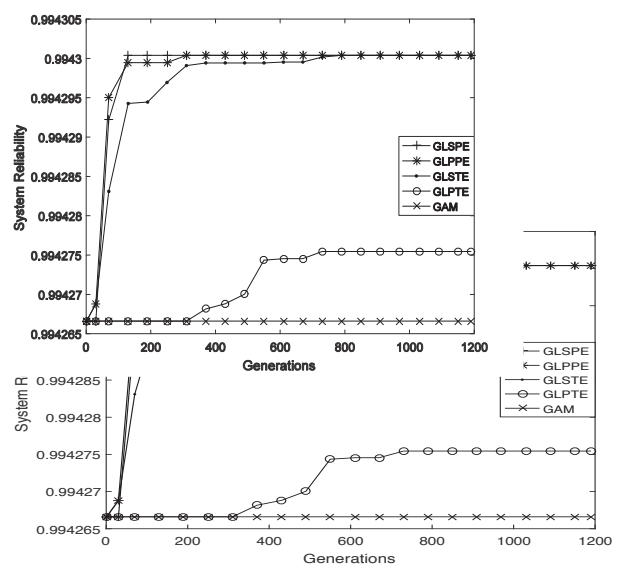

(d). Alternate component reliability 
Fig. 4. The evolution of the current best solutions for 1200 generations

As shown in Fig. 4, the GLSPE, GLPPE, GLSTE, and GLPTE use the BI-based local search in directing the solutions. Thus, they are more effective and always generate the better solutions than the GAM. Further, the GLSPE, GLPPE, and GLSTE perform better than the GLPTE and GAM for the tested instances. Section 5.2 confirms these observations through much more comprehensive numerical testing.

In Figs. 4(a), 4(c), and 4(d), the current best solutions from the GAM remains unchanged for the 1200 generations, and the similar result for the GLPTE is observed in Figs. 4(a) and 4(c). It is because for the five tested genetic algorithms, the initial generation includes the solution from the BITSM, which can be the best solution for many generations. It also implies that the solutions obtained from the GAM, GLSPE, GLPPE, GLSTE and GLPTE cannot be worse than the solution from the BITSM.

\subsection{Comparisons of the BITSM, GAM, GLSPE, GLPPE, GLSTE, and GLPTE}

To evaluate the BITSM, GAM, GLSPE, GLPPE, GLSTE, and GLPTE, we use these six methods with the same setting of parameters in Section 4.5 to solve the 3600 instances in the 72 trails as described in Section 2.3. Tables $3-6$ present the main results of comparing these methods for the four cases of component reliability: Low, High, Alternate, and Arbitrary, respectively. The meanings of the first nine columns in Tables $3-6$ are similar to the corresponding columns in Table 2. For example, columns "GLSPE $>\backslash<$ GAM" give two numbers separated by "" in each cell, which is associated with a trail of 50 instances. The first (second) number in a cell is the number of instances within the trail of 50 instances, for which the solution from the GLSPE is strictly better (worse) than the solution from the GAM. The number of instances for which the two methods obtain the same solutions equals 50 minus the two numbers in the cell. For example, among the 50 instances in the trial associated with the $\mathrm{Lin} / \mathrm{Con} / 4 / 40: \mathrm{G}$ system and low component reliability in Table 3, there are 47 instances for which the GLSPE obtains better solutions than the GAM, two instances for which the GLSPE obtains worse solutions than the GAM, and one instance for which the GLSPE and the GAM obtain the same solution. Therefore, an algorithm, say A, performs better (worse) than another algorithm, say B, for a trail if in the associated cell in columns " $\mathrm{A}>\mathrm{C}<\mathrm{B}$ ", the first number is larger (smaller) than the second number.

Table 3. Comparisons of the BITSM, GAM, GLSPE, GLPPE, GLSTE and GLPTE for the case of low component

\begin{tabular}{|c|c|c|c|c|c|c|c|c|c|c|c|c|c|c|}
\hline \multirow{5}{*}{$\begin{array}{r}\text { Syste } \\
\mathrm{ms}\end{array}$} & & & & & & & I.te & & & & & & & \\
\hline & \multirow{4}{*}{$\begin{array}{c}\text { GLSP } \\
\text { E } \\
>1< \\
\\
\text { GAM }\end{array}$} & \multirow{4}{*}{$\begin{array}{c}\text { GLPP } \\
\text { E } \\
>1< \\
\text { GAM }\end{array}$} & \multirow{4}{*}{$\begin{array}{c}\text { GLST } \\
\mathrm{E} \\
>1< \\
\text { GAM }\end{array}$} & \multirow{4}{*}{$\begin{array}{c}\text { GLPT } \\
\mathrm{E} \\
>1< \\
\text { GAM }\end{array}$} & \multirow{4}{*}{\begin{tabular}{|c} 
GLSP \\
$\mathrm{E}$ \\
$>\mathrm{k}$ \\
\\
GLPP \\
$\mathrm{E}$ \\
\end{tabular}} & \multirow{2}{*}{$\begin{array}{c}\text { GLSP } \\
\text { E } \\
>K<\end{array}$} & \multirow{2}{*}{$\begin{array}{c}\text { GLSP } \\
\mathrm{E} \\
>k\end{array}$} & \multirow{2}{*}{$\begin{array}{c}\text { GLSP } \\
\text { E } \\
>\end{array}$} & \multicolumn{6}{|c|}{ Computation time (seconds) } \\
\hline & & & & & & & & & \multirow{3}{*}{$\begin{array}{c}\text { BITS } \\
\text { M }\end{array}$} & \multirow{3}{*}{\multicolumn{2}{|c|}{$\begin{array}{c}\text { GLSP } \\
\text { E }\end{array}$}} & \multicolumn{3}{|c|}{$\begin{array}{c}\text { GLST } \\
\text { E }\end{array}$} \\
\hline & & & & & & GLST & GLPT & BITS & & & & G & & GLPT \\
\hline & & & & & & E & E & $\mathrm{M}$ & & & & E & & E \\
\hline $4 / 20: \mathrm{F}$ & $17 \backslash 0$ & $14 \backslash 0$ & $16 \backslash 0$ & $5 \backslash 0$ & $8 \backslash 2$ & $3 \backslash 1$ & $14 \backslash 0$ & 17 & 0.004 & 0.04 & 0.08 & 0.08 & 0.06 & 0.07 \\
\hline $5 / 20: F$ & $16 \backslash 0$ & $10 \backslash 0$ & $10 \backslash 1$ & $2 \backslash 1$ & $7 \backslash 0$ & $7 \backslash 0$ & $14 \backslash 0$ & 16 & 0.002 & 0.03 & 0.07 & 0.08 & 0.05 & 0.05 \\
\hline $\begin{array}{r}10 / 20: \\
F\end{array}$ & $3 \backslash 0$ & $3 \backslash 0$ & $1 \backslash 0$ & $2 \backslash 0$ & $0 \backslash 0$ & $2 \backslash 0$ & $1 \backslash 0$ & 6 & 0.001 & 0.03 & 0.06 & 0.04 & 0.03 & 0.02 \\
\hline 4/40:F & $43 \backslash 1$ & $44 \backslash 1$ & $46 \backslash 0$ & $21 \backslash 3$ & $17 \backslash 11$ & $20 \backslash 10$ & $41 \backslash 0$ & 44 & 0.013 & 1.06 & 4.19 & 5.21 & 2.39 & 3.65 \\
\hline $5 / 40: F$ & $47 \backslash 0$ & $45 \backslash 0$ & $45 \backslash 0$ & $15 \backslash 5$ & $19 \backslash 11$ & $24 \backslash 12$ & $44 \backslash 1$ & 47 & 0.012 & 1.14 & 3.90 & 4.70 & 2.34 & 3.87 \\
\hline $\begin{array}{r}\text { 10/40: } \\
F\end{array}$ & $38 \backslash 0$ & $29 \backslash 4$ & $29 \backslash 2$ & $16 \backslash 10$ & $27 \backslash 5$ & $22 \backslash 6$ & $36 \backslash 2$ & 42 & 0.008 & 0.86 & 2.13 & 3.57 & 1.54 & 1.62 \\
\hline 4/60:F & $48 \backslash 0$ & $48 \backslash 0$ & $49 \backslash 0$ & $18 \backslash 3$ & $33 \backslash 15$ & $22 \backslash 21$ & $48 \backslash 0$ & 48 & 0.034 & 1.03 & 9.68 & 12. & 4.8 & 7.76 \\
\hline $5 / 60: F$ & $49 \backslash 0$ & $50 \backslash 0$ & $48 \backslash 0$ & $15 \backslash 2$ & $23 \backslash 27$ & $25 \backslash 24$ & $49 \backslash 0$ & 49 & 0.030 & 1.41 & 10.47 & 12.25 & 4.36 & 8.98 \\
\hline $\begin{array}{r}\text { 10/60: } \\
F\end{array}$ & $46 \backslash 2$ & $33 \backslash 8$ & $42 \backslash 3$ & $16 \backslash 11$ & $36 \backslash 12$ & $27 \backslash 20$ & $44 \backslash 2$ & 46 & 0.022 & 2.00 & 7.63 & 10.52 & 3.67 & 5.39 \\
\hline $4 / 20: \mathrm{G}$ & $37 \backslash 0$ & $38 \backslash 0$ & $40 \backslash 0$ & $20 \backslash 8$ & $12 \backslash 6$ & $12 \backslash 7$ & $34 \backslash 1$ & 41 & 0.002 & 0.03 & 0.07 & 0.08 & 0.06 & 0.06 \\
\hline $5 / 20: \mathrm{G}$ & $27 \backslash 1$ & $27 \backslash 1$ & $21 \backslash 0$ & $10 \backslash 6$ & $5 \backslash 9$ & $12 \backslash 7$ & $24 \backslash 2$ & 31 & 0.002 & 0.03 & 0.0 & 0. & 0.05 & 0.05 \\
\hline 10/20: & $2 \backslash 1$ & $2 \backslash 0$ & $2 \backslash 1$ & $2 \backslash 3$ & $0 \backslash 1$ & $1 \backslash 1$ & $3 \backslash 1$ & 10 & 0.001 & 0.03 & 0.05 & 0.03 & 0.02 & 0.01 \\
\hline
\end{tabular}




\begin{tabular}{|c|c|c|c|c|c|c|c|c|c|c|c|c|c|c|}
\hline $\bar{G}$ & & & & & & & & & & & & & & \\
\hline 4/40:G & $47 \backslash 2$ & $47 \backslash 2$ & $46 \backslash 1$ & $33 \backslash 6$ & $21 \backslash 18$ & $31 \backslash 8$ & $46 \backslash 2$ & 49 & 0.009 & 0.92 & 2.94 & 4.16 & 2.56 & 3.02 \\
\hline $5 / 40: \mathrm{G}$ & $46 \backslash 1$ & $46 \backslash 1$ & $46 \backslash 1$ & $30 \backslash 8$ & $16 \backslash 21$ & $20 \backslash 15$ & $41 \backslash 5$ & 50 & 0.007 & 0.92 & 3.22 & 3.57 & 2.64 & 2.95 \\
\hline $\begin{array}{r}\text { 10/40: } \\
\mathrm{G}\end{array}$ & $26 \backslash 0$ & $25 \backslash 0$ & $25 \backslash 0$ & $9 \backslash 4$ & $2 \backslash 3$ & $4 \backslash 1$ & $26 \backslash 0$ & 32 & 0.003 & 0.45 & 1.83 & 2.05 & 0.90 & 1.20 \\
\hline $4 / 60: G$ & $50 \backslash 0$ & $49 \backslash 0$ & $47 \backslash 2$ & $39 \backslash 8$ & $27 \backslash 19$ & $20 \backslash 21$ & $46 \backslash 4$ & 50 & 0.022 & 0.96 & 6.82 & 8.24 & 5.40 & 5.83 \\
\hline $5 / 60: \mathrm{G}$ & $46 \backslash 1$ & $47 \backslash 0$ & $47 \backslash 0$ & $32 \backslash 4$ & $23 \backslash 17$ & $21 \backslash 15$ & $44 \backslash 2$ & 47 & 0.017 & 1.10 & 6.46 & 6.54 & 5.41 & 4.37 \\
\hline $\begin{array}{r}\text { 10/60: } \\
\mathrm{G}\end{array}$ & $44 \backslash 0$ & $43 \backslash 0$ & $38 \backslash 0$ & $14 \backslash 10$ & $9 \backslash 7$ & $12 \backslash 6$ & $37 \backslash 3$ & 45 & 0.009 & 1.15 & 3.13 & 3.56 & 3.46 & 1.78 \\
\hline
\end{tabular}

Table 4. Comparisons of the BITSM, GAM, GLSPE, GLPPE, GLSTE and GLPTE for the case of high component reliability

\begin{tabular}{|c|c|c|c|c|c|c|c|c|c|c|c|c|c|c|}
\hline \multirow{3}{*}{$\begin{array}{r}\text { Syste } \\
\mathrm{ms}\end{array}$} & \multirow{3}{*}{$\begin{array}{c}\text { GLSP } \\
\mathrm{E} \\
>1< \\
\text { GAM }\end{array}$} & \multirow{2}{*}{$\begin{array}{c}\text { GLPP } \\
\text { E } \\
>k<\end{array}$} & \multirow{2}{*}{$\begin{array}{c}\text { GLST } \\
\text { E } \\
>k<\end{array}$} & \multirow{2}{*}{$\begin{array}{c}\text { GLPT } \\
\text { E } \\
>k<\end{array}$} & \multirow{2}{*}{$\begin{array}{c}\text { GLSP } \\
E \\
>k\end{array}$} & \multirow{2}{*}{$\begin{array}{c}\text { GLSP } \\
\text { E } \\
>k\end{array}$} & \multirow{2}{*}{$\begin{array}{c}\text { GLSP } \\
\mathrm{E} \\
>\mathrm{k}<\end{array}$} & \multirow{2}{*}{$\begin{array}{c}\text { GLSP } \\
\text { E } \\
>\end{array}$} & \multicolumn{6}{|c|}{ Computation time (seconds) } \\
\hline & & & & & & & & & $\begin{array}{c}\text { BITS } \\
\text { M }\end{array}$ & & $\begin{array}{c}\text { GLSP } \\
\text { E }\end{array}$ & & $\begin{array}{c}\text { GLST } \\
\text { E }\end{array}$ & \\
\hline & & GAM & GAM & GAM & $\begin{array}{c}\text { GLPP } \\
\text { E }\end{array}$ & $\begin{array}{c}\text { GLST } \\
\text { E }\end{array}$ & $\begin{array}{c}\text { GLPT } \\
\text { E }\end{array}$ & $\begin{array}{c}\text { BITS } \\
\text { M }\end{array}$ & & $\begin{array}{r}\text { GA } \\
\mathrm{M}\end{array}$ & & $\begin{array}{r}\text { GLPP } \\
\mathrm{E} \\
\end{array}$ & & $\begin{array}{r}\text { GLPT } \\
\text { E }\end{array}$ \\
\hline $4 / 20: F$ & $\begin{array}{r}28 \backslash \\
0\end{array}$ & $\begin{array}{r}22 \backslash \\
1\end{array}$ & $\begin{array}{r}26 \backslash \\
0\end{array}$ & $4 \backslash 4$ & $\begin{array}{r}10 \backslash \\
1\end{array}$ & $6 \backslash 4$ & $\begin{array}{r}27 \backslash \\
0\end{array}$ & 28 & 0.003 & 0.04 & 0.07 & 0.09 & 0.05 & 0.07 \\
\hline $5 / 20: F$ & $20 \backslash 0$ & $17 \backslash 0$ & $13 \backslash 0$ & $3 \backslash 2$ & $5 \backslash 0$ & $12 \backslash 1$ & $20 \backslash 0$ & 21 & 0.002 & 0.04 & 0.07 & 0.09 & 0.05 & 0.06 \\
\hline $\begin{array}{r}\text { 10/20: } \\
\mathrm{F}\end{array}$ & $4 \backslash 0$ & $4 \backslash 0$ & $3 \backslash 4$ & $3 \backslash 1$ & $0 \backslash 1$ & $5 \backslash 0$ & $2 \backslash 0$ & 8 & 0.001 & 0.03 & 0.07 & 0.05 & 0.04 & 0.03 \\
\hline 4/40:F & $48 \backslash 0$ & $50 \backslash 0$ & $48 \backslash 0$ & $24 \backslash 5$ & $22 \backslash 13$ & $26 \backslash 13$ & $47 \backslash 1$ & 48 & 0.013 & 1.11 & 4.05 & 3 & 2.56 & 4.48 \\
\hline $5 / 40: F$ & $49 \backslash 0$ & $48 \backslash 0$ & $49 \backslash 0$ & $25 \backslash 4$ & $22 \backslash 15$ & $21 \backslash 18$ & $49 \backslash 0$ & 49 & 0.012 & 1.12 & 3.95 & 5.09 & 3.03 & 4.27 \\
\hline $\begin{array}{r}\text { 10/40: } \\
\mathrm{F}\end{array}$ & $44 \backslash 2$ & $34 \backslash 9$ & $37 \backslash 7$ & $22 \backslash 15$ & $31 \backslash 6$ & $27 \backslash 10$ & $43 \backslash 3$ & 47 & 0.011 & 1.35 & 1.57 & 1.98 & 1.84 & 1.93 \\
\hline 4/60:F & $48 \backslash 0$ & $49 \backslash 0$ & $49 \backslash 0$ & $22 \backslash 3$ & $24 \backslash 24$ & $23 \backslash 26$ & $48 \backslash 0$ & 48 & 37 & 1.15 & 10.33 & & 5.82 & 9.53 \\
\hline 5/60:F & $49 \backslash 0$ & $50 \backslash 0$ & $50 \backslash 0$ & $21 \backslash 5$ & $25 \backslash 25$ & $31 \backslash 18$ & $49 \backslash 0$ & 49 & 0.033 & 1.16 & 11.51 & 77 & 4.93 & 10.00 \\
\hline $\begin{array}{r}\text { 10/60: } \\
\mathrm{F}\end{array}$ & $48 \backslash 0$ & $46 \backslash 3$ & $49 \backslash 0$ & $29 \backslash 7$ & $39 \backslash 11$ & $21 \backslash 27$ & $47 \backslash 2$ & 48 & 0.029 & 1.50 & 9.59 & 10.02 & 4.68 & 6.37 \\
\hline 4/20:G & $43 \backslash 1$ & $44 \backslash 2$ & $43 \backslash 2$ & $29 \backslash 10$ & $14 \backslash 13$ & $17 \backslash 15$ & $41 \backslash 3$ & 48 & 0.002 & 0.0 & $0.0^{\prime}$ & 0 & 0.07 & 0.06 \\
\hline 5/20:G & $27 \backslash 0$ & $29 \backslash 1$ & $22 \backslash 2$ & $8 \backslash 11$ & $2 \backslash 8$ & $11 \backslash 2$ & $28 \backslash 1$ & 33 & 0.001 & 0.03 & 0.06 & 0.07 & 0.05 & 0.05 \\
\hline $\begin{array}{r}10 / 20: \\
\mathrm{G}\end{array}$ & $1 \backslash 2$ & $0 \backslash 0$ & $1 \backslash 1$ & $0 \backslash 0$ & $1 \backslash 2$ & $0 \backslash 1$ & $1 \backslash 2$ & 5 & 0.001 & 0.03 & 0.05 & 0.03 & 0.03 & 0.01 \\
\hline 4/40:G & $47 \backslash 1$ & $48 \backslash 1$ & $45 \backslash 2$ & $32 \backslash 15$ & $22 \backslash 21$ & $27 \backslash 16$ & $40 \backslash 4$ & 50 & 0.009 & 1.29 & 2.90 & 3.6 & 2.65 & 2.26 \\
\hline 5/40:G & $44 \backslash 3$ & $48 \backslash 1$ & $49 \backslash 0$ & $29 \backslash 11$ & $18 \backslash 26$ & $24 \backslash 17$ & $44 \backslash 3$ & 50 & 0.007 & 1.53 & 3.66 & 4.13 & 2.81 & 2.85 \\
\hline $\begin{array}{r}\text { 10/40: } \\
\mathrm{G}\end{array}$ & $23 \backslash 3$ & $24 \backslash 2$ & $20 \backslash 3$ & $4 \backslash 19$ & $2 \backslash 5$ & $8 \backslash 2$ & $33 \backslash 2$ & 37 & 0.003 & 0.91 & 1.94 & 2.10 & 0.75 & 1.05 \\
\hline 4/60:G & $10 \backslash 1$ & 711 & 170 & 010 & 311 & 4 & 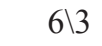 & 10 & 0.015 & 0.1 & 0.8 & & 1.12 & 1.94 \\
\hline 5/60:G & $50 \backslash 0$ & $49 \backslash 0$ & $48 \backslash 1$ & $38 \backslash 11$ & $14 \backslash 34$ & $26 \backslash 22$ & $50 \backslash 0$ & 50 & 0.018 & 1.10 & 6.34 & 7.99 & 4.92 & 6.08 \\
\hline $\begin{array}{r}10 / 60: \\
\mathrm{G} \\
\end{array}$ & $43 \backslash 1$ & $45 \backslash 0$ & $38 \backslash 1$ & $16 \backslash 14$ & $10 \backslash 16$ & $13 \backslash 12$ & $44 \backslash 0$ & 47 & 0.008 & 1.35 & 4.52 & 3.99 & 2.54 & 2.40 \\
\hline
\end{tabular}

Table 5. Comparisons of the BITSM, GAM, GLSPE, GLPPE, GLSTE and GLPTE for the case of alternate component reliability

\begin{tabular}{|c|c|c|c|c|c|c|c|c|c|c|c|c|c|c|}
\hline \multirow{3}{*}{ Systems } & \multirow{3}{*}{$\begin{array}{c}\text { GLSPE } \\
>l< \\
\text { GAM }\end{array}$} & \multirow{3}{*}{$\begin{array}{c}\text { GLPPE } \\
>\backslash< \\
\text { GAM }\end{array}$} & \multirow{3}{*}{$\begin{array}{c}\text { GLSTE } \\
><< \\
\text { GAM }\end{array}$} & \multirow{3}{*}{$\begin{array}{c}\text { GLPTE } \\
>\mid< \\
\text { GAM }\end{array}$} & \multirow{3}{*}{$\begin{array}{c}\text { GLSPE } \\
><< \\
\text { GLPPE }\end{array}$} & \multirow{3}{*}{$\begin{array}{c}\text { GLSPE } \\
>\backslash< \\
\text { GLSTE }\end{array}$} & \multirow{3}{*}{$\begin{array}{c}\text { GLSPE } \\
>\backslash< \\
\text { GLPTE }\end{array}$} & \multirow{3}{*}{$\begin{array}{c}\text { GLSPE } \\
> \\
\text { BITSM }\end{array}$} & \multicolumn{6}{|c|}{ Computation time (seconds) } \\
\hline & & & & & & & & & \multirow[t]{2}{*}{ BITSM } & & \multirow{2}{*}{ GLSPE } & \multicolumn{3}{|c|}{ GLSTE } \\
\hline & & & & & & & & & & GAM & & GLPPE & & GLPTE \\
\hline $4 / 20: F$ & $22 \backslash 0$ & $22 \backslash 1$ & $23 \backslash 0$ & $11 \backslash 0$ & $9 \backslash 4$ & $6 \backslash 6$ & $18 \backslash 2$ & 23 & 0.003 & 0.04 & 0.08 & 0.09 & 0.06 & 0.07 \\
\hline $5 / 20: F$ & $23 \backslash 0$ & $18 \backslash 0$ & $21 \backslash 0$ & $4 \backslash 0$ & $12 \backslash 1$ & $7 \backslash 1$ & $23 \backslash 0$ & 23 & 0.002 & 0.03 & 0.07 & 0.08 & 0.05 & 0.06 \\
\hline 10/20:F & $0 \backslash 0$ & $0 \backslash 0$ & $0 \backslash 3$ & $0 \backslash 1$ & $0 \backslash 0$ & $3 \backslash 0$ & $1 \backslash 0$ & 5 & 0.001 & 0.03 & 0.05 & 0.05 & 0.04 & 0.02 \\
\hline 4/40:F & $48 \backslash 0$ & $45 \backslash 0$ & $46 \backslash 0$ & $21 \backslash 3$ & $20 \backslash 15$ & $23 \backslash 9$ & $47 \backslash 0$ & 48 & 0.014 & 1.26 & 4.06 & 5.82 & 2.79 & 4.09 \\
\hline
\end{tabular}




\begin{tabular}{|c|c|c|c|c|c|c|c|c|c|c|c|c|c|c|}
\hline $5 / 40: F$ & $49 \backslash 0$ & $48 \backslash 1$ & $49 \backslash 0$ & $30 \backslash 4$ & $25 \backslash 15$ & $20 \backslash 16$ & $48 \backslash 0$ & 49 & 0.012 & 1.10 & 4.05 & 4.48 & 3.11 & 4.26 \\
\hline 10/40:F & $41 \backslash 1$ & $33 \backslash 6$ & $32 \backslash 4$ & $17 \backslash 14$ & $27 \backslash 5$ & $21 \backslash 6$ & $41 \backslash 3$ & 45 & 0.009 & 1.48 & 2.53 & 3.97 & 2.10 & 2.81 \\
\hline $4 / 60: F$ & $48 \backslash 0$ & $48 \backslash 0$ & $47 \backslash 0$ & $28 \backslash 0$ & $25 \backslash 23$ & $26 \backslash 23$ & $48 \backslash 0$ & 49 & 0.039 & 1.12 & 9.75 & 12.39 & 4.89 & 8.83 \\
\hline $5 / 60: F$ & $50 \backslash 0$ & $48 \backslash 1$ & $47 \backslash 0$ & $18 \backslash 4$ & $32 \backslash 18$ & $33 \backslash 17$ & $50 \backslash 0$ & 50 & 0.035 & 1.13 & 11.34 & 14.87 & 5.36 & 8.67 \\
\hline 10/60:F & $48 \backslash 1$ & $47 \backslash 2$ & $48 \backslash 1$ & $14 \backslash 16$ & $37 \backslash 13$ & $33 \backslash 16$ & $48 \backslash 1$ & 48 & 0.023 & 1.77 & 9.81 & 11.09 & 3.67 & 6.48 \\
\hline $4 / 20: G$ & $40 \backslash 0$ & $40 \backslash 0$ & $40 \backslash 0$ & $17 \backslash 6$ & $12 \backslash 13$ & $9 \backslash 14$ & $36 \backslash 3$ & 41 & 0.002 & 0.04 & 0.07 & 0.08 & 0.06 & 0.06 \\
\hline $5 / 20: \mathrm{G}$ & $33 \backslash 1$ & $33 \backslash 2$ & $30 \backslash 0$ & $12 \backslash 13$ & $5 \backslash 2$ & $9 \backslash 2$ & $32 \backslash 1$ & 36 & 0.002 & 0.04 & 0.07 & 0.06 & 0.05 & 0.05 \\
\hline 10/20:G & $1 \backslash 0$ & $1 \backslash 0$ & $1 \backslash 1$ & $1 \backslash 2$ & $0 \backslash 0$ & $1 \backslash 0$ & $2 \backslash 0$ & 8 & 0.001 & 0.03 & 0.05 & 0.03 & 0.03 & 0.01 \\
\hline 4/40:G & $50 \backslash 0$ & $48 \backslash 1$ & $48 \backslash 0$ & $30 \backslash 12$ & $23 \backslash 22$ & $22 \backslash 17$ & $46 \backslash 3$ & 50 & 0.008 & 1.46 & 4.01 & 4.39 & 3.13 & 3.47 \\
\hline $5 / 40: G$ & $47 \backslash 1$ & $47 \backslash 1$ & $45 \backslash 1$ & $30 \backslash 9$ & $17 \backslash 22$ & $21 \backslash 12$ & $46 \backslash 3$ & 50 & 0.008 & 1.19 & 3.30 & 4.18 & 2.41 & 2.57 \\
\hline 10/40:G & $16 \backslash 0$ & $15 \backslash 0$ & $12 \backslash 1$ & $5 \backslash 6$ & $1 \backslash 0$ & $6 \backslash 1$ & $17 \backslash 0$ & 27 & 0.004 & 0.67 & 2.04 & 2.34 & 0.95 & 1.37 \\
\hline 4/60:G & $50 \backslash 0$ & $50 \backslash 0$ & $50 \backslash 0$ & $41 \backslash 8$ & $25 \backslash 24$ & $26 \backslash 24$ & $49 \backslash 1$ & 50 & 0.021 & 1.01 & 7.81 & 9.19 & 4.80 & 6.51 \\
\hline $5 / 60: G$ & $49 \backslash 1$ & $50 \backslash 0$ & $49 \backslash 1$ & $40 \backslash 7$ & $17 \backslash 31$ & $26 \backslash 20$ & $45 \backslash 4$ & 50 & 0.019 & 1.16 & 5.28 & 5.97 & 4.35 & 6.73 \\
\hline 10/60:G & $44 \backslash 2$ & $45 \backslash 0$ & $41 \backslash 0$ & $17 \backslash 12$ & $14 \backslash 18$ & $19 \backslash 10$ & $44 \backslash 3$ & 47 & 0.009 & 1.62 & 3.50 & 3.40 & 3.10 & 2.03 \\
\hline
\end{tabular}

Table 6. Comparisons of the BITSM, GAM, GLSPE, GLPPE, GLSTE and GLPTE for the case of arbitrary components reliability

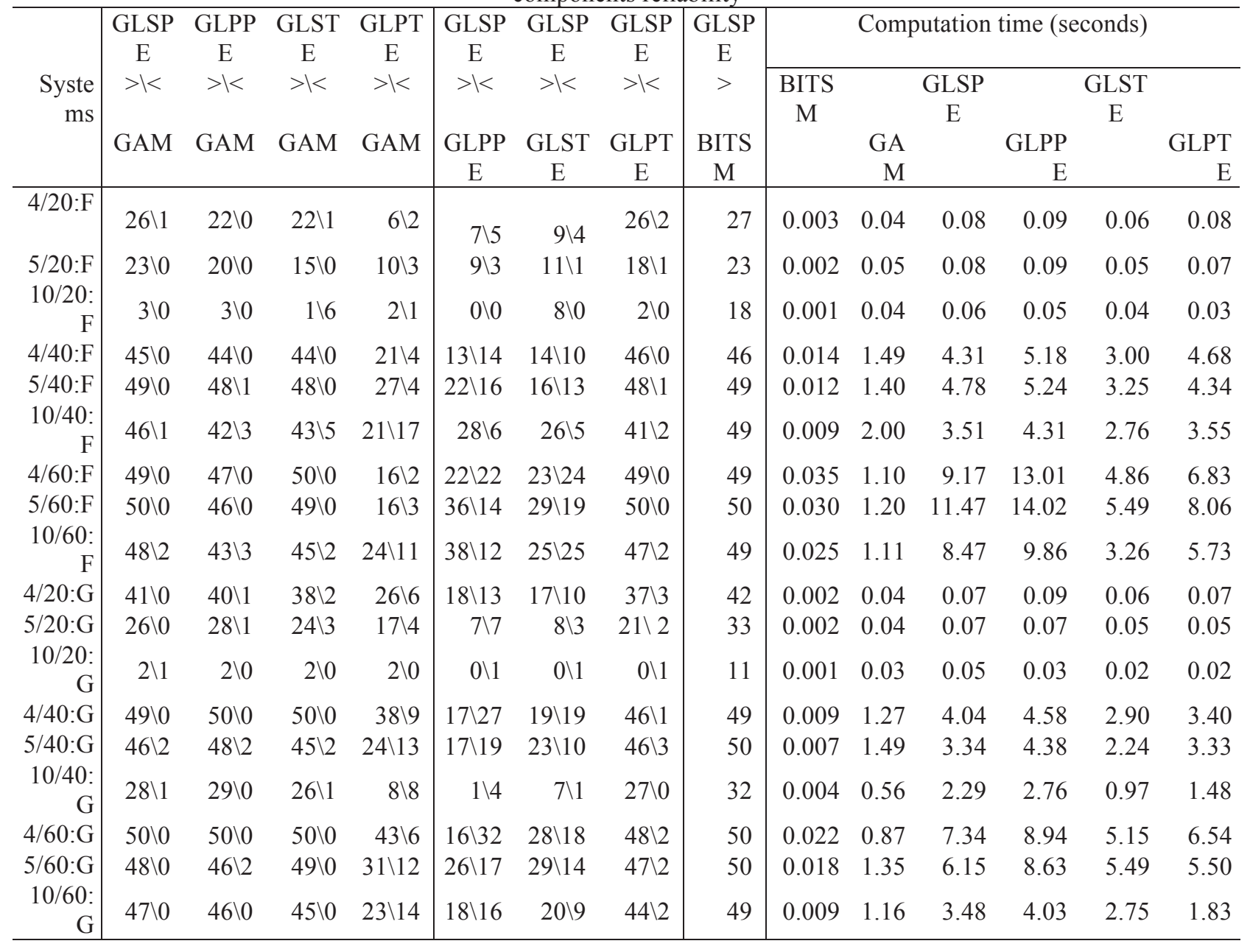

In Tables $3-6$, the second to fifth columns compare the BI-based genetic local search methods (i.e., the GLSPE, GLPPE, GLSTE, and GLPTE) to the GAM. The second numbers in the cells in columns 
"GLSPE $>\mid<$ GAM", "GLPPE $>\mid<$ GAM", and "GLSTE $>\mid<$ GAM" are less than 10. It implies that the GLSPE, GLPPE, and GLSTE can obtain the solutions no worse than the GAM for more than 40 over 50 instances for all the 72 trials. By columns "GLPTE $><$ GAM", the GLPTE can obtain the better solutions than the GAM for more instances in 64 out of 72 trails. Thus, the BI-based genetic local search methods, especially the GLSPE, GLPPE, and GLSTE, are superior to the genetic algorithm without the BI-based local search, i.e., the GAM. It demonstrates the effectiveness of the BI-based local search under the evolutionary mechanism for addressing the MCAP.

Further, the sixth to eighth columns in Tables $3-6$ compare the GLSPE to the GLPPE, GLSTE, and GLPTE. The GLSPE can obtain the better solutions than the GLPPE for more instances in 50 out of 72 trails (see columns "GLSPE $><<$ GLPPE"). The GLSPE can obtain the better solutions than the GLSTE for more instances in 65 out of 72 trails (see columns "GLSPE $><$ GLSTE"). The GLSPE can obtain the solutions no worse than the GLPTE for more than 44 instances in all the 72 trails (see the second numbers in the cells in columns "GLSPE > K GLPTE"). Thus, the GLSPE is superior to the GLPPE, GLSTE, and GLPTE, especially the GLSTE and GLPTE.

We also conduct the numerical testing for the revised SPE, revised PPE, revised STE, and revised PTE, which is same as the numerical testing for the SPE, PPE, STE, and PTE in Section 3.3. By the revised algorithms, it means that Step B.1.3 is removed from the SPE and STE, and Step B.2 is removed from the PPE and PTE, as illustrated in Section 4.6. As one main result in point (b) in Section 3.3, the SPE, PPE, STE, and PTE obtain 2034, 4549, 1268, and 765 best solutions, respectively. Correspondingly, the revised SPE, revised PPE, revised STE, and revised PTE obtain 3221, 2648, 1907, and 780 best solutions, respectively. That is, the revised SPE is superior to the other three local search methods. This result is very consistent to the result in the preceding paragraph. It implies that a good BI-based local search may be also good at its use in the genetic local search.

As illustrated in Section 5.1, the five genetic algorithms include the solution from the BITSM in their initial generations, and thus their solutions are no worse than the BISTM. Based on the proceeding analysis, the GLSPE is the best one among the five genetic algorithms. Thus, columns "GLSPE > BISTM" in Tables $3-6$ record the numbers of instances for which the GLSPE can improve the solutions from the BITSM. It is shown that the GLSPE can improve the solutions from the BISTM for 5 to 50 instances among the 50 instances in a trial and can improve the solutions for more than 40 instances in 47 out of 72 trials, especially for the trials in the large-size systems with large value of $n_{\alpha}=n / k(M=k)$. It is because for the small-size systems and the small values of $n_{\alpha}$, the solution space ( $\left.\alpha=1 M n \alpha !\right)$ is relatively small, and thus the BISTM can obtain the exact optimal or super solutions and leave limited room to the GLSPE to improve the solutions. It demonstrates the strengths of the global search in combination with the BI over the local search.

Note that the revised STE and revised PTE need three components for the three-way exchange, and thus the GLSTE and GLPTE reduce to the GAM for addressing the Lin/Con/10/20:F(G) systems that have two components in each type. For the similar reason, the GLSPE and GLPPE (i.e., the pair-wise exchange) also work similarly as the GAM for addressing the Lin/Con/10/20:F(G) systems. As shown in Tables 3 - 6, for the Lin/Con/10/20:F(G) systems, the GAM, GLSTE, GLPTE, GLPPE and GLSPE perform similarly, and the slight differences are due to the randomness of the genetic algorithms.

In Tables $3-6$, columns "computation time" record the average computation time for solving an instance in a trial. Over the tested instances, the BITSM takes 0.001 to 0.039 seconds, the GAM takes 0.03 to 2.00 seconds, the GLPTE takes 0.01 to 10.00 seconds, the GLSTE takes 0.02 to 5.82 seconds, the GLPPE takes 0.03 to 14.87 seconds, and the GLSPE takes 0.05 to 11.51 seconds. Based on these data, we observe and illustrate the following points about the computation time.

a. Clearly, the BITSM is the fastest method. It is the advantage of the local search method.

b. The GLSPE, GLPPE, GLSTE and GLPTE take more time than the GAM because of the local search.

c. The GLSPE and GLPPE take more time than the GLSTE and GLPTE because the revised SPE and revised PPE evaluate about $(n-k)$ pair-wise exchanges each time, while the revised STE and revised PTE evaluate about $(n / 2-k)$ three-way exchanges. 
d. For the GLSPE, GLPPE, GLSTE and GLPTE, the average computation time tends to increase with the number of components $(n)$ and the numbers of components in each type $\left(n_{\alpha}\right)$ because the solution space ( $\alpha=1 M n \alpha !)$ increases with these two factors. For a fixed $n$ values, the computation time decreases as $M(M=k)$ increases. An exception exists for the $\operatorname{Lin} / \mathrm{Con} / 4 / 60: \mathrm{G}$ system with high component reliability in Table 4, for which the system reliability is very close to 1 . Thus, the chromosomes have such closed fitness values that termination condition in Eq. (11) is satisfied within a few generations and the algorithms converge fast.

e. Overall, the five genetic local search methods are efficient.

\section{Conclusions and future research}

In this paper, we propose a new type of system reliability design problem, namely, the MCAP, and establish a nonlinear binary programming model for it. We propose and evaluate seventeen local search methods and five genetic-algorithm based global search methods. All the heuristics presented in this paper use the BI to direct the assignment of components and can be used to solve the MCAP in arbitrary systems. The comprehensive numerical experiments and the results demonstrate the effectiveness of the $\mathrm{BI}$ in designing the algorithms for the MCAP.

We present and compare the sequential manner and parallel manner of dealing with multiple types of components. The BITSM, which uses the parallel manner in both Stage A and Sage B, is proposed as the most efficient local search method. The GLSPE, which use the revised SPE as the local search method, is recommended as the most efficient global search method. An effective BI-based local search is important for the performance of the genetic local search algorithm. With more computational effort, the BI-based genetic local search is superior to the BI-based local search in terms of solution quality.

As future research, the heuristics in this paper can be modified to deal with the MCAP with dependent components if the BI of the dependent components can be calculated as in [8], but the calculations may be more complicated and the numerical testing deserves further investigation.

\section{Acknowledgements}

This work was supported in part by the National Science Foundation of China under grant NSFC\# 71571178 and Chinese Thousand Youth Talents Program, and the National Science Foundation under award \# CMMI-1633500.

\section{References}

[1] Chang GJ, Cui L, Huang FK. Reliabilities of Consecutive- $k$ Systems. Boston: Kluwer academic; 2000.

[2] Kuo W, Zhu X. Impotence Measures in Reliability, Risk, and Optimization: Principles and Applications. Hoboken: Wiley; 2012.

[3] Lin Y-K, Yeh C-T. Reliability optimization of component assignment problem for a multistate network in terms of minimal cuts. J Ind Manag Optim 2011;7:211-27. doi:10.3934/jimo.2011.7.211.

[4] Yeh C-T, Lin Y-K. Component allocation cost minimization for a multistate computer network subject to a reliability threshold using tabu search. J Ind Manag Optim 2015;12:141-67. doi:10.3934/jimo.2016.12.141.

[5] Lin Y-K, Yeh C-T. Maximal network reliability with optimal transmission line assignment for stochastic electric power networks via genetic algorithms. Appl Soft Comput 2011;11:2714-24. doi:10.1016/j.asoc.2010.11.002.

[6]Yao Q, Zhu X, Kuo W. A Birnbaum-importance based genetic local search algorithm for component assignment problems. Ann Oper Res 2014; 212:185-200. doi:10.1007/s10479-012-1223-1.

[7] Shingyochi K, Yamamoto H, Tsujimura Y, Akiba T. Proposal of simulated annealing algorithms for optimal arrangement in a circular consecutive-k-out-of-n:F system. Qual Technol Quant Manag 
2010; 7:395-405. doi:10.1080/16843703.2010.11673241.

[8] Shingyoch K, Yamamoto H, Tsujimura Y, Kambayashi Y. Improvement of ordinal representation scheme for solving optimal component arrangement problem of circular consecutive-k-out-of-n:F system. Qual Technol Quant Manag 2009; 6:11-22. doi:10.1080/16843703.2009.11673181.

[9] Yao Q, Zhu X, Kuo W. Heuristics for component assignment problems based on the Birnbaum importance. IIE Trans 2011; 43:633-46. doi:10.1080/0740817X.2010.532856.

[10] Lin FH, Kuo W. Reliability importance and invariant optimal allocation. J Heuristics 2002; 8:155-71. doi:10.1023/A:1017908523107.

[11] Natvig B, Huseby AB, Reistadbakk MO. Measures of component importance in repairable multistate systems - a numerical study. Reliab Eng Syst Saf 2011;96:1680-90. doi:10.1016/j.ress.2011.07.006.

[12] Vaurio JK. Importance measures in risk-informed decision making: Ranking, optimisation and configuration control. Reliab Eng Syst Saf 2011;96:1426-36. doi:10.1016/j.ress.2011.06.012.

[13] Contini S, Matuzas V. New methods to determine the importance measures of initiating and enabling events in fault tree analysis. Reliab Eng Syst Saf 2011;96:775-84. doi:10.1016/j.ress.2011.02.001.

[14] Vaurio JK. Importance measures for multi-phase missions. Reliab. Eng. Syst. Saf., vol. 96, 2011, p. 230-5. doi:10.1016/j.ress.2010.07.002.

[15] Espiritu JF, Coit DW, Prakash U. Component criticality importance measures for the power industry. Electr Power Syst Res 2007;77:407-20. doi:10.1016/j.epsr.2006.04.003.

[16] Bisanovic S, Samardzic M, Aganovic D. Application of Component Criticality Importance Measures in Design Scheme of Power Plants. Int J Electr Comput Eng 2016;6:63. doi:10.11591/ijece.v6i1.9061.

[17] Kuo W, Zhu X. Relations and generalizations of importance measures in reliability. IEEE Trans Reliab 2012; 61:659-74. doi:10.1109/TR.2012.2208302.

[18] Kuo W, Zhu X. Some recent advances on importance measures in reliability. IEEE Trans Reliab 2012; 61:344-60. doi:10.1109/TR.2012.2194196.

[19] Zhu X, Kuo W. Importance measures in reliability and mathematical programming. Ann Oper Res 2014;212:241-67. doi:10.1007/s10479-012-1127-0.

[20] Birnbaum ZW. On the importance of different components in a multicomponent system. In PR Krishnaiah editor. Multivariate analysis II. New York: Academic Press; 1969. pp. 581-592

[21] Kontoleon JM. Optimum link allocation of fixed topology networks. IEEE Trans Reliab 1979; R28:145-7. doi:10.1109/TR.1979.5220527.

[22] Zuo M, Kuo W. Design and performance analysis of consecutive-k-out-of-n structure. Nav Res Logist 1990; 37:203-30. doi:10.1002/1520-6750(199004)37:2<203::AID-NAV 3220370203>3.0. $\mathrm{CO} ; 2-\mathrm{X}$.

[23] Sadjadi SJ, Soltani R. An efficient heuristic versus a robust hybrid meta-heuristic for general framework of serial-parallel redundancy problem. Reliab Eng Syst Saf 2009; 94:1703-10. doi:10.1016/j.ress.2009.05.003.

[24] Feizabadi M, Jahromi AE. A new model for reliability optimization of series-parallel systems with non-homogeneous components. Reliab Eng Syst Saf 2017;157:101-12. doi:10.1016/j.ress.2016.08.023.

[25] Cai Z, Si S, Sun S, Li C. Optimization of linear consecutive-k-out-of-n system with a Birnbaum importance-based genetic algorithm. Reliab Eng Syst Saf 2016; 152:248-58. doi:10.1016/j.ress.2016.03.016.

[26] Chiang DT, Niu S-C. Reliability of consecutive-k-out-of-n:F System. IEEE Trans Reliab 1981; R-30:87-9. doi:10.1109/TR.1981.5220981.

[27] Kuo W, Zhang W, Zuo M. A consecutive-k-out-of-n:G system: the mirror image of a consecutive-k-out-of-n:F system. IEEE Trans Reliab 1990; 39:244-53. doi:10.1109/24.55888.

[28] Lim MH, Yuan Y, Omatu S. Efficient genetic algorithms using simple genes exchange local search policy for the quadratic assignment problem. Comput Optim Appl 2000; 15:249-68. doi: 10.1023/A:1008743718053.

[29] Goldberg DE. Genetic algorithms in search, optimization, and machine learning. Reading: Addison-Wesley; 1989. 